\title{
Does the Reaction of Cyclopropyl Acid Chlorides and Imines to Form 1,3-0xazin-4-enone heterocycles Proceed via a Ketene or an $N$-Acyl- Iminium Mechanism?
}

\author{
Alexander J. Craig, Andrew P. Cording, Anna L. Garden, and Bill C. Hawkins* \\ Department of Chemistry, University of Otago, PO Box 56, Dunedin, New Zealand \\ bhawkins@chemistry.otago.ac.nz
}

Supporting Information

\section{Contents}

Imine computational data 


\section{Iminium pathway}

$1 a$ - Imine plus acid chloride

SCF Energy: -1016.502812 a.u.

$\begin{array}{llll}\mathrm{C} & -2.62918 & 0.01365 & -0.00789 \\ \mathrm{C} & -1.20027 & -0.43583 & 0.07283 \\ \mathrm{C} & -0.64226 & -0.99961 & 1.36041 \\ \mathrm{C} & -0.88581 & -1.91159 & 0.20826 \\ \mathrm{H} & -1.31277 & -1.02584 & 2.21185 \\ \mathrm{H} & 0.39355 & -0.75091 & 1.56162 \\ \mathrm{H} & -0.01650 & -2.24444 & -0.34922 \\ \mathrm{H} & -1.72112 & -2.60199 & 0.22931 \\ \mathrm{O} & -2.95295 & 0.83824 & -0.84385 \\ \mathrm{C} & -3.62640 & -0.55047 & 0.97214 \\ \mathrm{H} & -4.62828 & -0.42770 & 0.56189 \\ \mathrm{H} & -3.44679 & -1.60013 & 1.21188 \\ \mathrm{H} & -3.55536 & 0.02267 & 1.90284 \\ \mathrm{C} & 3.24359 & 0.02619 & 0.39546 \\ \mathrm{H} & 4.22603 & 0.02899 & -0.10214 \\ \mathrm{~N} & 2.33308 & -0.77595 & 0.02314 \\ \mathrm{C} & -0.31145 & 0.37331 & -0.82576 \\ \mathrm{O} & 0.08639 & 0.07405 & -1.90324 \\ \mathrm{C} & 3.06185 & 1.00285 & 1.51386 \\ \mathrm{H} & 3.17280 & 2.02618 & 1.14022 \\ \mathrm{H} & 2.07648 & 0.89661 & 1.97012 \\ \mathrm{H} & 3.83578 & 0.84920 & 2.27263 \\ \mathrm{C} & 2.64749 & -1.68769 & -1.06574 \\ \mathrm{H} & 3.66299 & -1.55177 & -1.46213 \\ \mathrm{H} & 2.54038 & -2.71729 & -0.71015 \\ \mathrm{H} & 1.92589 & -1.53969 & -1.87478 \\ \mathrm{Cl} & 0.08838 & 1.98734 & -0.14650\end{array}$




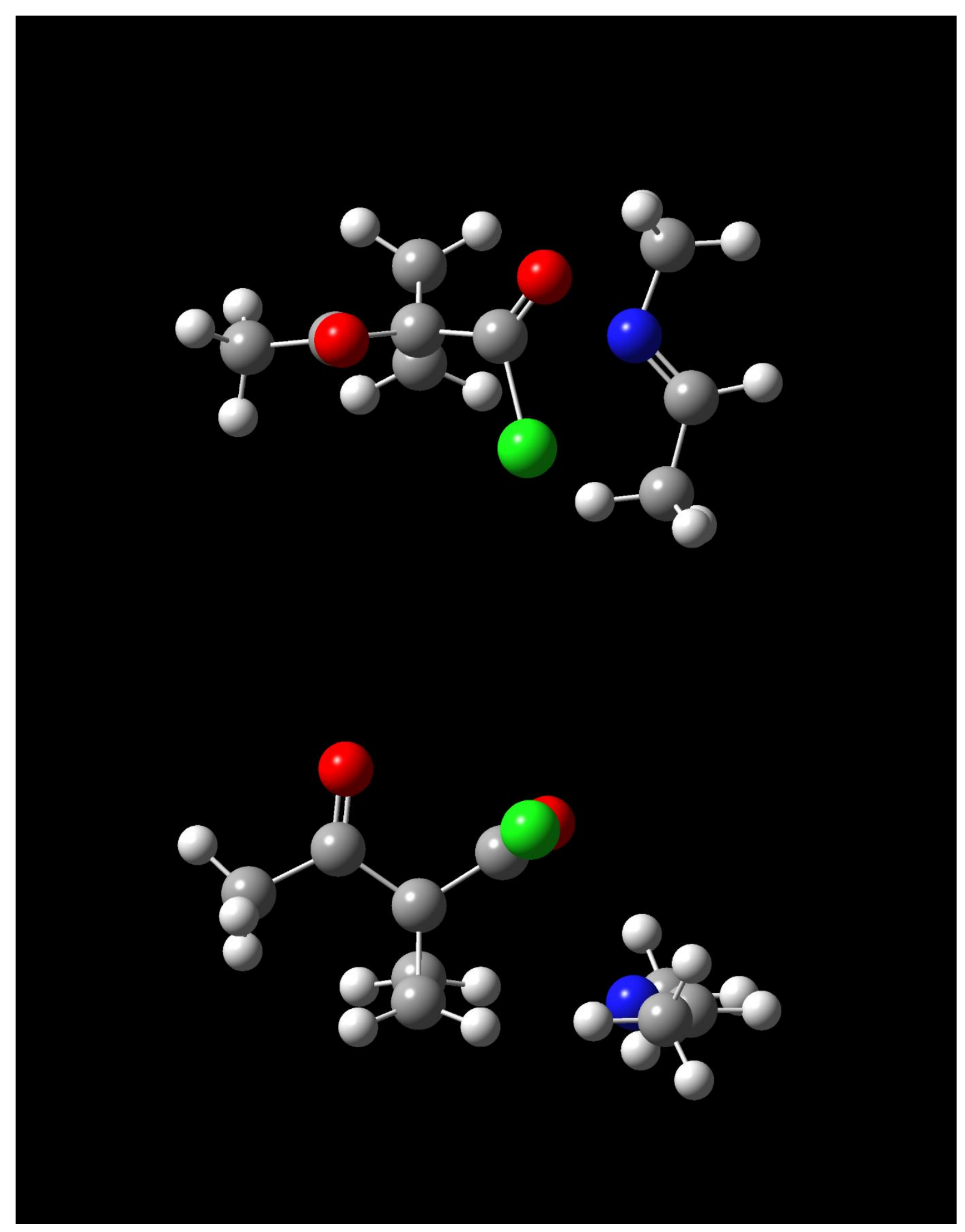


$1 b$ - Tetrahedral intermediate transition state

SCF Energy: -1016.484459 a.u.

$\begin{array}{llll}\mathrm{C} & -2.234340 & 0.14447 & -0.03642 \\ \mathrm{C} & -0.874805 & -0.50659 & -0.16116 \\ \mathrm{C} & -0.592332 & -1.76043 & 0.62909 \\ \mathrm{C} & -0.915700 & -1.87843 & -0.81948 \\ \mathrm{H} & -1.329930 & -2.06038 & 1.36346 \\ \mathrm{H} & 0.428265 & -1.96542 & 0.91456 \\ \mathrm{H} & -0.099815 & -2.09834 & -1.49990 \\ \mathrm{H} & -1.882095 & -2.25873 & -1.13182 \\ \mathrm{O} & -2.467990 & 1.21267 & -0.56406 \\ \mathrm{C} & -3.304657 & -0.57875 & 0.75084 \\ \mathrm{H} & -4.258485 & -0.08505 & 0.56953 \\ \mathrm{H} & -3.382166 & -1.63609 & 0.48548 \\ \mathrm{H} & -3.065423 & -0.52028 & 1.81778 \\ \mathrm{C} & 2.47316 & -0.51601 & 0.74234 \\ \mathrm{H} & 3.51598 & -0.83461 & 0.65488 \\ \mathrm{~N} & 1.83504 & -0.31701 & -0.34485 \\ \mathrm{C} & 0.18964 & 0.44475 & -0.68488 \\ \mathrm{O} & 0.21958 & 0.83208 & -1.82369 \\ \mathrm{C} & 1.96676 & -0.36967 & 2.13410 \\ \mathrm{H} & 2.52822 & 0.43994 & 2.61265 \\ \mathrm{H} & 0.90575 & -0.14817 & 2.19609 \\ \mathrm{H} & 2.20311 & -1.28851 & 2.68095 \\ \mathrm{C} & 2.52840 & -0.48742 & -1.62096 \\ \mathrm{H} & 3.52996 & -0.88694 & -1.45787 \\ \mathrm{H} & 1.95578 & -1.16548 & -2.25586 \\ \mathrm{H} & 2.58279 & 0.47841 & -2.12345 \\ \mathrm{Cl} & 0.35843 & 1.93761 & 0.65227\end{array}$




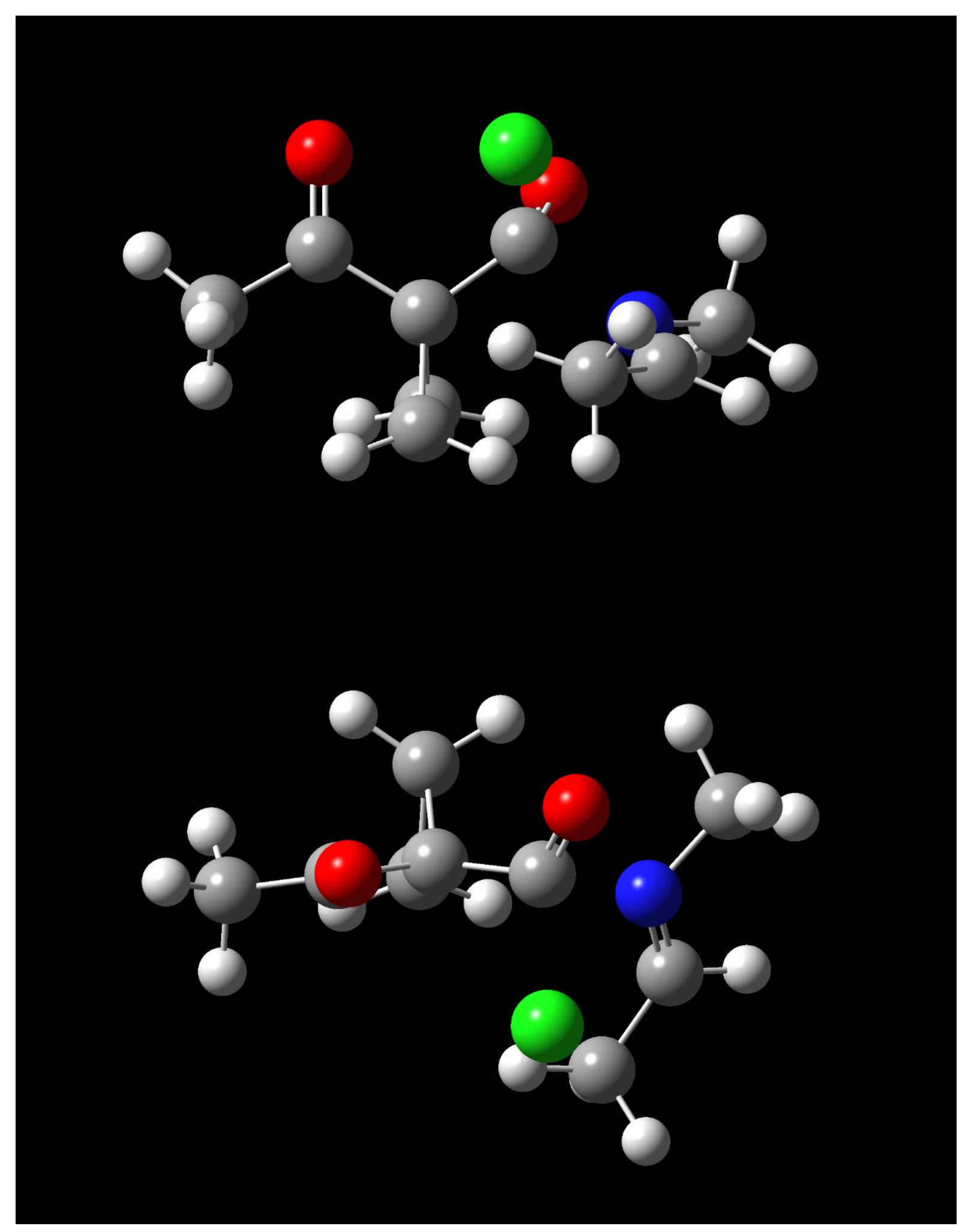


$1 c 1$ - Iminium intermediate with chloride frozen 10 Angstrom away

SCF Energy: -1016.491737a.u.

$\begin{array}{llll}\mathrm{C} & 2.83396 & -1.17782 & -0.44741 \\ \mathrm{C} & 2.13288 & -0.23677 & 0.46534 \\ \mathrm{C} & 1.33816 & -0.72146 & 1.66598 \\ \mathrm{C} & 2.65279 & -0.09974 & 1.90239 \\ \mathrm{H} & 1.23964 & -1.7973 & 1.80909 \\ \mathrm{H} & 0.45715 & -0.11892 & 1.92377 \\ \mathrm{H} & 2.65322 & 0.89604 & 2.30505 \\ \mathrm{H} & 3.50002 & -0.70439 & 2.18975 \\ \mathrm{O} & 2.94687 & -0.90942 & -1.61524 \\ \mathrm{C} & 3.31713 & -2.49635 & 0.08948 \\ \mathrm{H} & 4.15298 & -2.83427 & -0.5251 \\ \mathrm{H} & 3.5932 & -2.48894 & 1.15133 \\ \mathrm{H} & 2.49796 & -3.21066 & -0.03355 \\ \mathrm{C} & -0.68057 & 0.7133 & -0.59391 \\ \mathrm{H} & -1.67399 & 1.16026 & -0.51082 \\ \mathrm{~N} & 0.28988 & 1.44968 & -0.16929 \\ \mathrm{C} & 1.74688 & 1.03757 & -0.20955 \\ \mathrm{O} & 2.46769 & 1.82761 & -0.74729 \\ \mathrm{C} & -0.5394 & -0.67294 & -1.17258 \\ \mathrm{H} & -1.2874 & -0.7708 & -1.98319 \\ \mathrm{H} & 0.46079 & -0.87142 & -1.55803 \\ \mathrm{H} & -0.81132 & -1.40282 & -0.40442 \\ \mathrm{C} & 0.12469 & 2.83058 & 0.34514 \\ \mathrm{H} & -0.93176 & 3.09402 & 0.29614 \\ \mathrm{H} & 0.4857 & 2.93022 & 1.36561 \\ \mathrm{H} & 0.71777 & 3.49353 & -0.28795 \\ \mathrm{Cl} & -8.11521 & -0.58389 & 0.12225\end{array}$




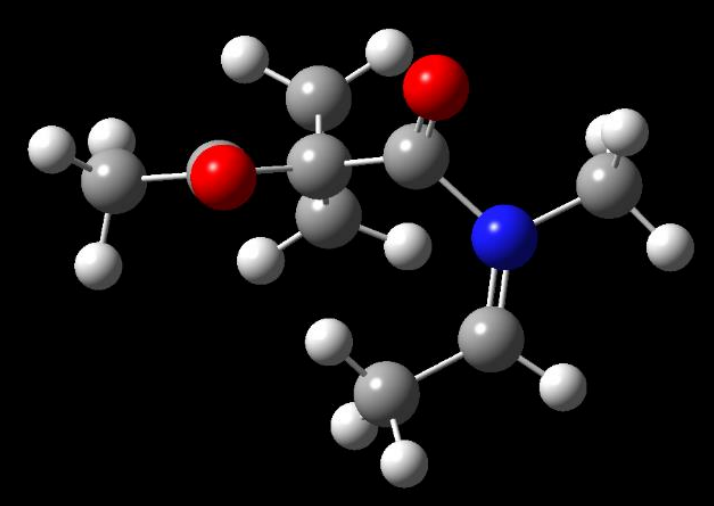

e

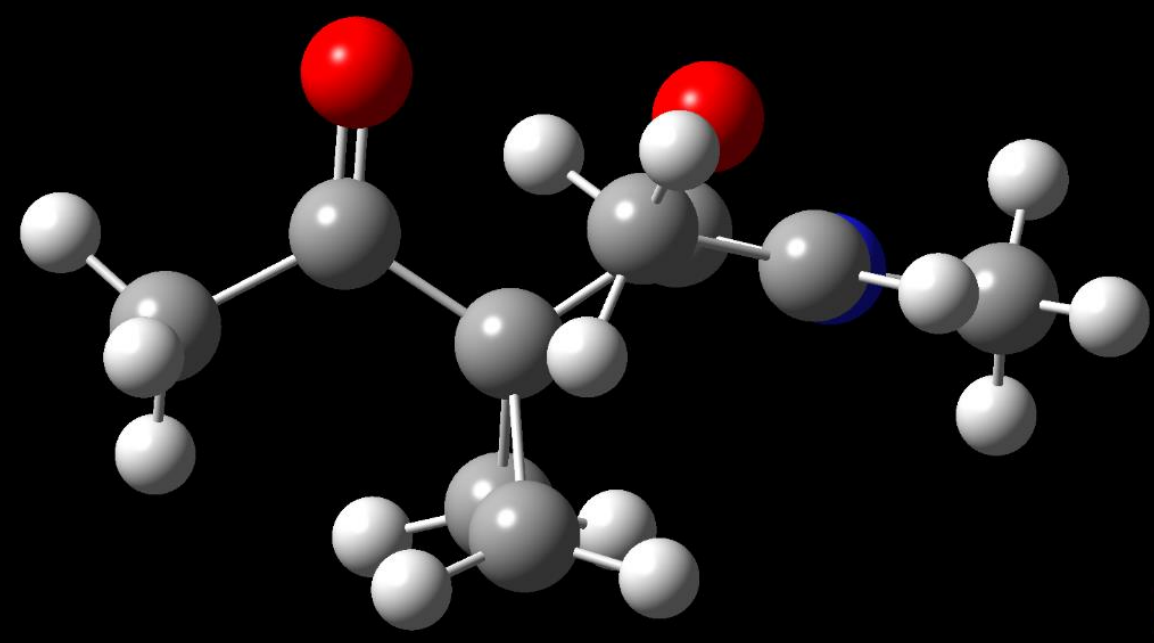


$1 c 2$ - Iminium intermediate no chloride

SCF Energy: -556.161205 a.u.

$\begin{array}{llll}\mathrm{C} & 1.14679 & 1.09539 & -0.24092 \\ \mathrm{C} & 0.97146 & -0.39431 & -0.16210 \\ \mathrm{C} & 1.96508 & -1.27452 & -0.91547 \\ \mathrm{C} & 1.98656 & -1.29544 & 0.55908 \\ \mathrm{H} & 2.74927 & -0.74890 & -1.44758 \\ \mathrm{H} & 1.52271 & -2.11554 & -1.43666 \\ \mathrm{H} & 1.57059 & -2.16011 & 1.06467 \\ \mathrm{H} & 2.77774 & -0.77967 & 1.09116 \\ \mathrm{O} & 0.20164 & 1.78181 & -0.58823 \\ \mathrm{C} & 2.48136 & 1.69236 & 0.11149 \\ \mathrm{H} & 2.52063 & 2.71389 & -0.26396 \\ \mathrm{H} & 2.58101 & 1.70831 & 1.20260 \\ \mathrm{H} & 3.31915 & 1.11676 & -0.28826 \\ \mathrm{C} & -2.38172 & 0.48911 & 0.16457 \\ \mathrm{H} & -3.03216 & 1.02280 & 0.85555 \\ \mathrm{~N} & -1.36755 & -0.09689 & 0.69173 \\ \mathrm{C} & -0.42156 & -0.90037 & -0.13272 \\ \mathrm{O} & -0.83322 & -1.91572 & -0.61028 \\ \mathrm{C} & -2.70579 & 0.51835 & -1.27375 \\ \mathrm{H} & -2.79732 & 1.57004 & -1.56328 \\ \mathrm{H} & -1.97372 & 0.02052 & -1.90491 \\ \mathrm{H} & -3.69208 & 0.06335 & -1.41255 \\ \mathrm{C} & -1.04516 & -0.03245 & 2.12896 \\ \mathrm{H} & -1.84694 & 0.49303 & 2.64368 \\ \mathrm{H} & -0.95149 & -1.04906 & 2.51252 \\ \mathrm{H} & -0.10406 & 0.50532 & 2.25817\end{array}$




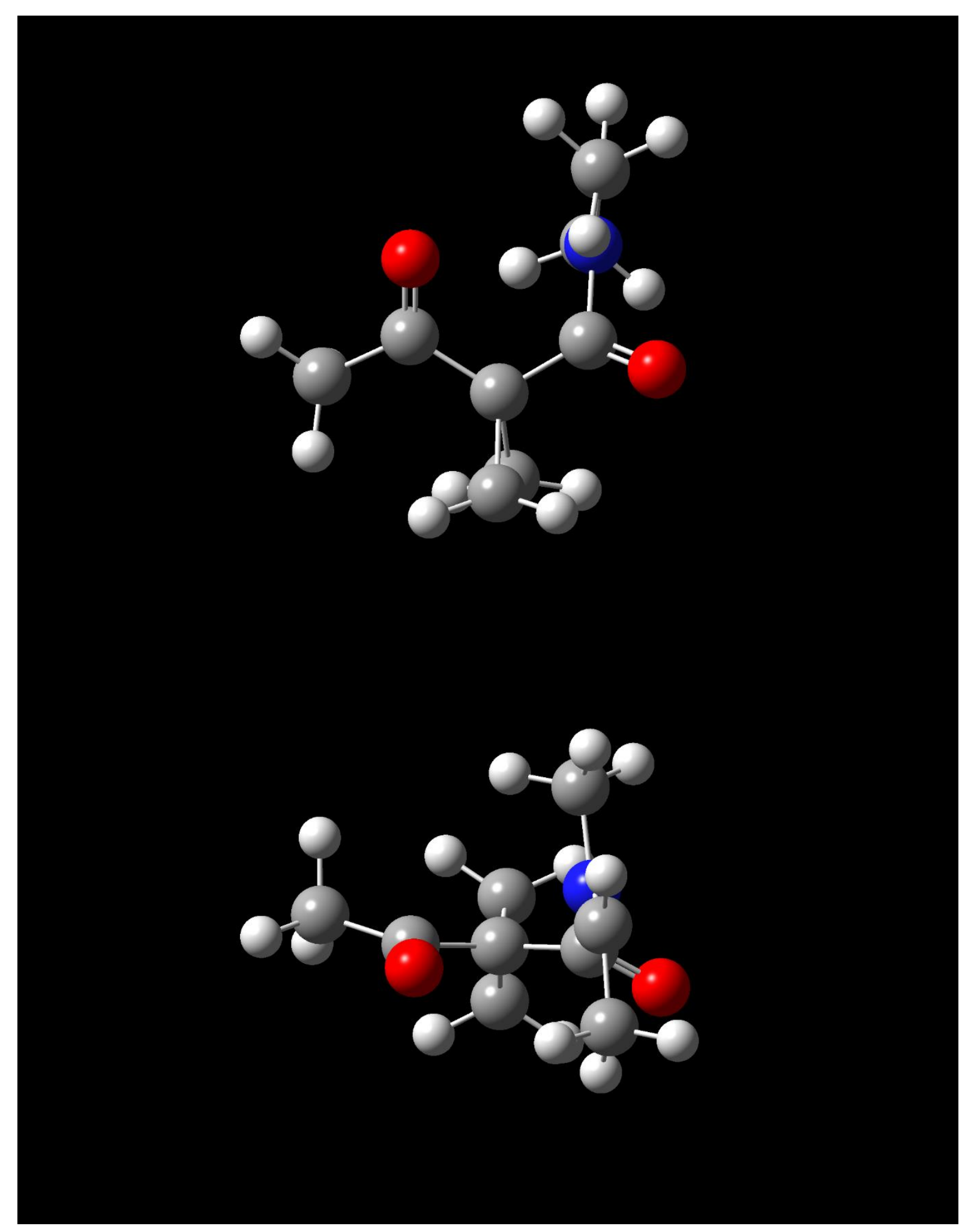


$1 d$-Oxazinone cation transition state

SCF Energy: -556.152236 a.u.

$\begin{array}{llll}\mathrm{C} & -1.04655 & 0.94336 & 0.42245 \\ \mathrm{C} & -0.82517 & -0.46531 & -0.04383 \\ \mathrm{C} & -1.92269 & -1.50759 & 0.20809 \\ \mathrm{C} & -1.77233 & -0.98683 & -1.15414 \\ \mathrm{H} & -2.76292 & -1.18320 & 0.80853 \\ \mathrm{H} & -1.54162 & -2.49931 & 0.41936 \\ \mathrm{H} & -1.27492 & -1.60430 & -1.89466 \\ \mathrm{H} & -2.48809 & -0.27045 & -1.54172 \\ \mathrm{O} & -0.14456 & 1.78244 & 0.44044 \\ \mathrm{C} & -2.41306 & 1.34947 & 0.89027 \\ \mathrm{H} & -2.45181 & 2.43471 & 0.96205 \\ \mathrm{H} & -3.20043 & 0.99086 & 0.22404 \\ \mathrm{H} & -2.58804 & 0.91432 & 1.87955 \\ \mathrm{C} & 1.58586 & 1.06842 & -0.36628 \\ \mathrm{H} & 2.25243 & 1.80729 & 0.07181 \\ \mathrm{~N} & 1.61456 & -0.12132 & 0.19761 \\ \mathrm{C} & 0.54634 & -1.05463 & -0.07029 \\ \mathrm{O} & 0.78242 & -2.22754 & -0.17796 \\ \mathrm{C} & 1.16185 & 1.29049 & -1.77470 \\ \mathrm{H} & 2.06557 & 1.15597 & -2.38065 \\ \mathrm{H} & 0.40996 & 0.58186 & -2.12328 \\ \mathrm{H} & 0.81271 & 2.31375 & -1.90613 \\ \mathrm{C} & 2.43084 & -0.42520 & 1.38779 \\ \mathrm{H} & 3.23641 & 0.30309 & 1.45178 \\ \mathrm{H} & 1.80881 & -0.38359 & 2.28449 \\ \mathrm{H} & 2.84657 & -1.42410 & 1.26556\end{array}$



1e-Oxazinone cation

SCF Energy: -556.174474 a.u.

$\begin{array}{llll}\mathrm{C} & -1.08901 & -0.88757 & -0.33317 \\ \mathrm{C} & -0.91134 & 0.48888 & 0.03552 \\ \mathrm{C} & -2.08232 & 1.46807 & -0.19246 \\ \mathrm{C} & -1.80966 & 1.05615 & 1.17872 \\ \mathrm{H} & -2.94695 & 1.07083 & -0.70999 \\ \mathrm{H} & -1.72838 & 2.44157 & -0.51128 \\ \mathrm{H} & -1.25377 & 1.72845 & 1.82335 \\ \mathrm{H} & -2.45592 & 0.33961 & 1.67229 \\ \mathrm{O} & -0.07730 & -1.65577 & -0.47311 \\ \mathrm{C} & -2.39741 & -1.51584 & -0.62203 \\ \mathrm{H} & -2.32030 & -2.59778 & -0.53063 \\ \mathrm{H} & -3.17902 & -1.12654 & 0.03200 \\ \mathrm{H} & -2.66693 & -1.26127 & -1.65460 \\ \mathrm{C} & 1.26397 & -1.21310 & -0.07447 \\ \mathrm{H} & 1.92429 & -1.80395 & -0.70809 \\ \mathrm{~N} & 1.43848 & 0.18054 & -0.40108 \\ \mathrm{C} & 0.46631 & 1.08017 & -0.09793 \\ \mathrm{O} & 0.64748 & 2.28387 & -0.02141 \\ \mathrm{C} & 1.43504 & -1.55043 & 1.39278 \\ \mathrm{H} & 2.45090 & -1.28433 & 1.69359 \\ \mathrm{H} & 0.72788 & -0.98517 & 2.00660 \\ \mathrm{H} & 1.29019 & -2.61971 & 1.55399 \\ \mathrm{C} & 2.80681 & 0.65465 & -0.60502 \\ \mathrm{H} & 3.30581 & 0.84986 & 0.34856 \\ \mathrm{H} & 3.35874 & -0.10305 & -1.16203 \\ \mathrm{H} & 2.76839 & 1.57717 & -1.18184\end{array}$




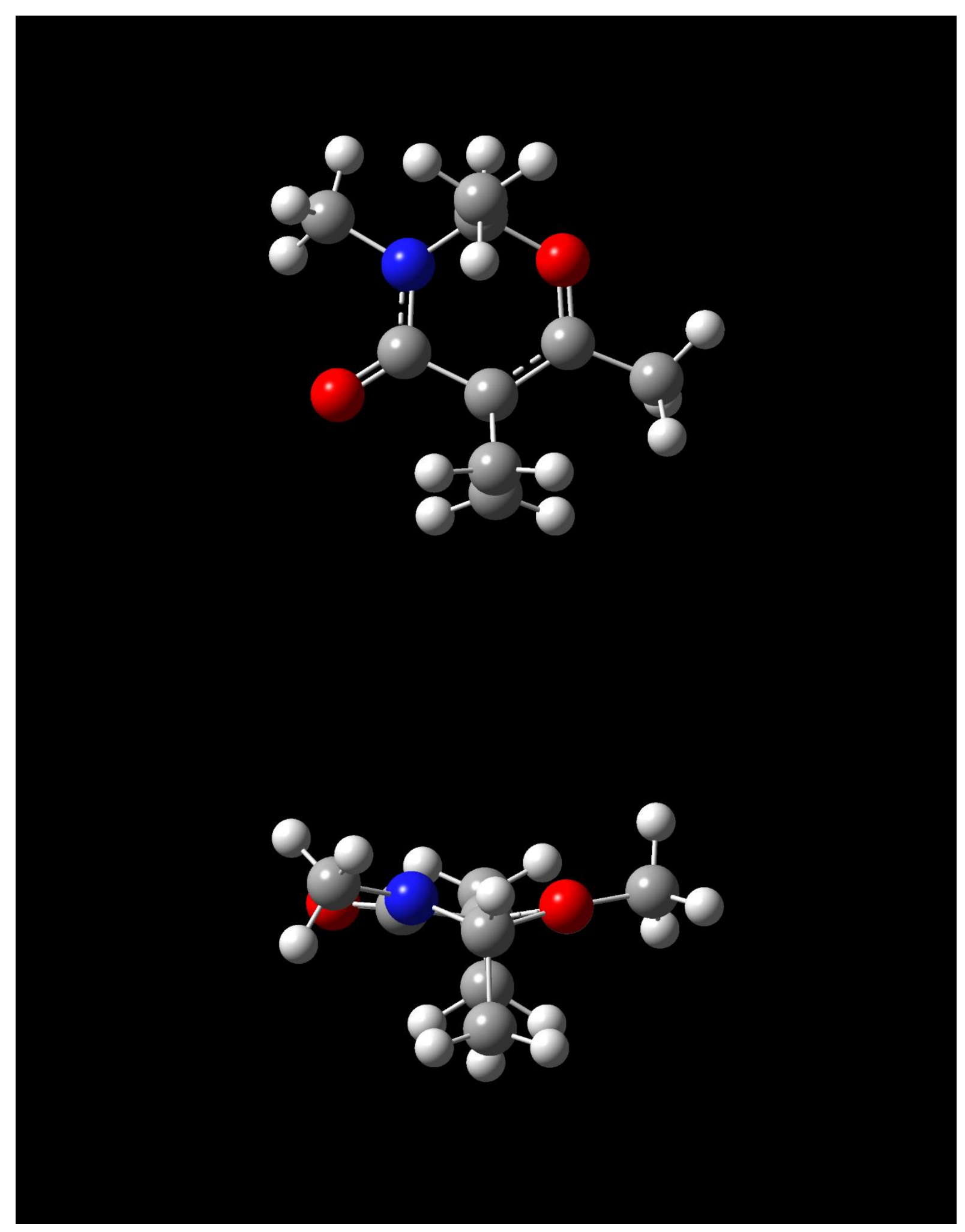


1f-Oxazinone cation plus chloride

SCF Energy: -1016.528172 a.u.

$\begin{array}{llll}\mathrm{C} & -0.37679 & -0.49621 & 0.78184 \\ \mathrm{C} & -0.10888 & 0.82602 & 0.27133 \\ \mathrm{C} & -1.16495 & 1.43115 & -0.72239 \\ \mathrm{C} & -1.03256 & 1.98755 & 0.62467 \\ \mathrm{H} & -2.02040 & 0.79694 & -0.94839 \\ \mathrm{H} & -0.68754 & 1.94213 & -1.55181 \\ \mathrm{H} & -0.49228 & 2.91874 & 0.74863 \\ \mathrm{H} & -1.81711 & 1.77718 & 1.34225 \\ \mathrm{O} & 0.45227 & -1.44062 & 0.52925 \\ \mathrm{C} & -1.55055 & -0.86349 & 1.58507 \\ \mathrm{H} & -1.48722 & -1.90615 & 1.89058 \\ \mathrm{H} & -1.63493 & -0.20247 & 2.45129 \\ \mathrm{H} & -2.45115 & -0.71578 & 0.95700 \\ \mathrm{C} & 1.52365 & -1.17849 & -0.44973 \\ \mathrm{H} & 0.98990 & -1.11972 & -1.40618 \\ \mathrm{~N} & 2.14787 & 0.07834 & -0.11613 \\ \mathrm{C} & 1.33253 & 1.16669 & -0.00159 \\ \mathrm{O} & 1.71520 & 2.32386 & -0.04874 \\ \mathrm{Cl} & -4.14039 & -0.51851 & -0.72496 \\ \mathrm{C} & 2.43875 & -2.37239 & -0.39627 \\ \mathrm{H} & 3.20068 & -2.29475 & -1.17320 \\ \mathrm{H} & 2.91611 & -2.44974 & 0.58271 \\ \mathrm{H} & 1.84652 & -3.26899 & -0.58288 \\ \mathrm{C} & 3.57964 & 0.28072 & -0.33717 \\ \mathrm{H} & 4.14857 & -0.47551 & 0.20175 \\ \mathrm{H} & 3.82452 & 0.24315 & -1.40269 \\ \mathrm{H} & 3.83111 & 1.26598 & 0.04952\end{array}$




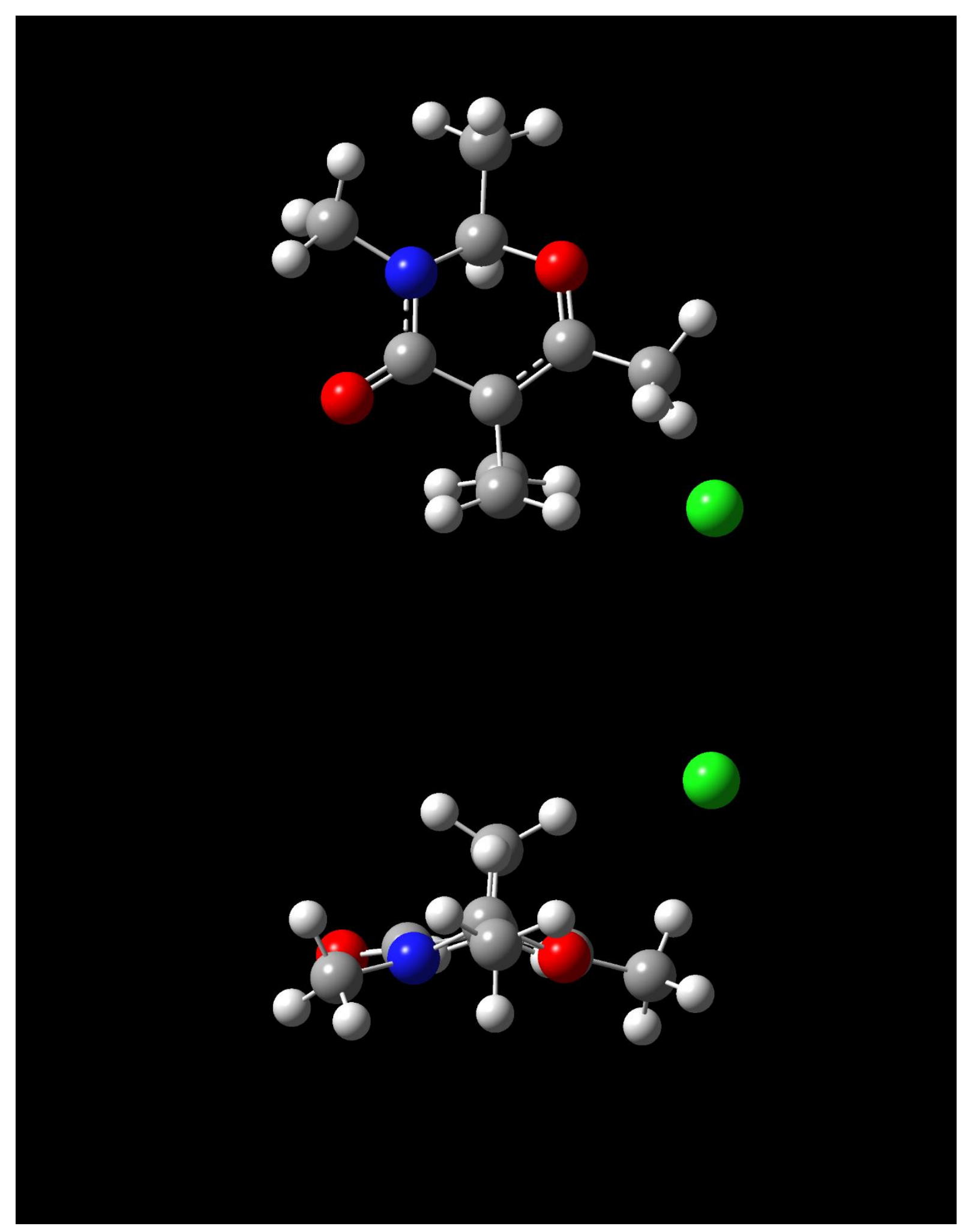


$1 f$-Oxazinone cation to oxazinone transition state

SCF Energy: -1016.511144 a.u.

$\begin{array}{llll}\mathrm{C} & 0.33034 & 1.39409 & 0.13027 \\ \mathrm{C} & -0.21329 & 0.17660 & 0.50804 \\ \mathrm{C} & -1.59692 & 0.04007 & 1.14843 \\ \mathrm{C} & -1.88220 & -0.01341 & -0.26147 \\ \mathrm{H} & -1.92923 & 0.91094 & 1.70425 \\ \mathrm{H} & -1.67458 & -0.90068 & 1.68553 \\ \mathrm{H} & -1.79696 & -0.95445 & -0.78858 \\ \mathrm{H} & -2.10808 & 0.87977 & -0.82549 \\ \mathrm{O} & 1.59487 & 1.47353 & -0.22373 \\ \mathrm{C} & -0.37918 & 2.69827 & 0.04046 \\ \mathrm{H} & 0.16680 & 3.43207 & 0.63838 \\ \mathrm{H} & -0.36045 & 3.03747 & -0.99998 \\ \mathrm{H} & -1.41020 & 2.64285 & 0.38357 \\ \mathrm{C} & 2.47311 & 0.40408 & 0.20570 \\ \mathrm{H} & 2.56502 & 0.52613 & 1.29442 \\ \mathrm{~N} & 1.86388 & -0.87415 & -0.11194 \\ \mathrm{C} & 0.57385 & -1.07381 & 0.29004 \\ \mathrm{O} & 0.04618 & -2.17488 & 0.38302 \\ \mathrm{Cl} & -4.45246 & -0.37258 & -0.46768 \\ \mathrm{C} & 3.79488 & 0.63866 & -0.48134 \\ \mathrm{H} & 4.54922 & -0.05408 & -0.10581 \\ \mathrm{H} & 3.69024 & 0.51943 & -1.56220 \\ \mathrm{H} & 4.12140 & 1.65578 & -0.26007 \\ \mathrm{C} & 2.72089 & -2.03626 & -0.33438 \\ \mathrm{H} & 3.35973 & -1.86705 & -1.20077 \\ \mathrm{H} & 3.33969 & -2.24362 & 0.54512 \\ \mathrm{H} & 2.07486 & -2.89061 & -0.52299\end{array}$




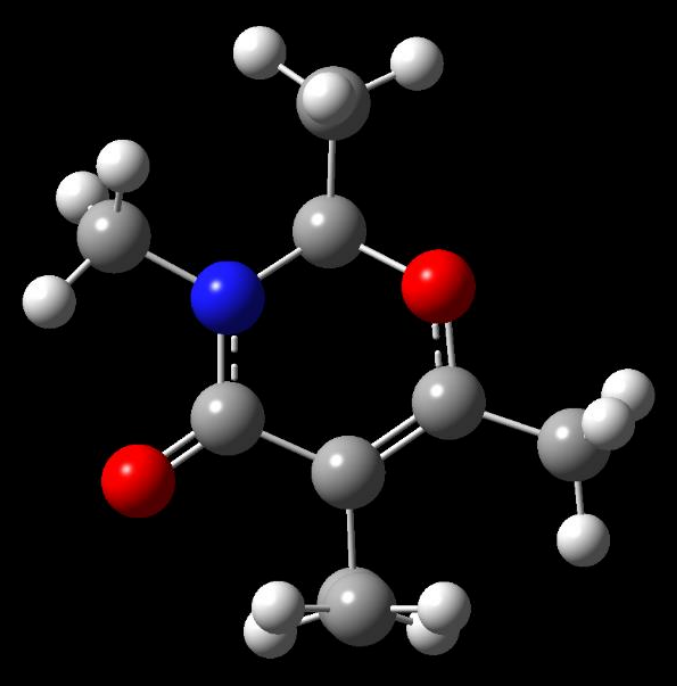

e

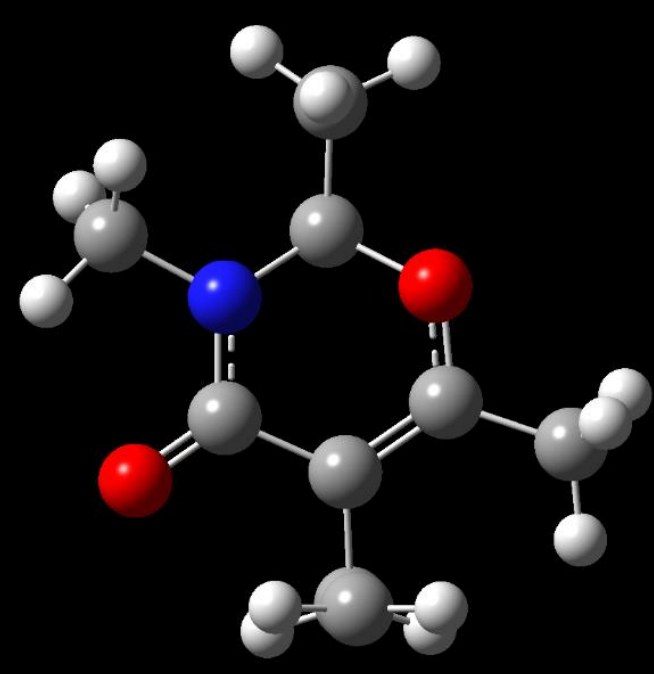

c 
$1 g$ - Oxazinone from iminium

SCF Energy: -1016.563976 a.u.

$\begin{array}{llll}\mathrm{C} & 0.38744 & 1.40499 & 0.04739 \\ \mathrm{C} & -0.21707 & 0.24071 & 0.37309 \\ \mathrm{C} & -1.67650 & 0.12051 & 0.72539 \\ \mathrm{C} & -2.50258 & -0.14094 & -0.52703 \\ \mathrm{H} & -2.03471 & 1.02261 & 1.22720 \\ \mathrm{H} & -1.80697 & -0.71735 & 1.41546 \\ \mathrm{H} & -2.18957 & -1.06352 & -1.01478 \\ \mathrm{H} & -2.44519 & 0.69012 & -1.23122 \\ \mathrm{O} & 1.71338 & 1.44671 & -0.23805 \\ \mathrm{C} & -0.24469 & 2.75029 & -0.08777 \\ \mathrm{H} & 0.15913 & 3.42908 & 0.66917 \\ \mathrm{H} & 0.00811 & 3.16167 & -1.06936 \\ \mathrm{H} & -1.32854 & 2.70717 & 0.00852 \\ \mathrm{C} & 2.46788 & 0.36876 & 0.31023 \\ \mathrm{H} & 2.40511 & 0.46779 & 1.40715 \\ \mathrm{~N} & 1.86144 & -0.88896 & -0.09935 \\ \mathrm{C} & 0.52191 & -1.02564 & 0.16101 \\ \mathrm{O} & -0.05468 & -2.11505 & 0.14656 \\ \mathrm{Cl} & -4.25323 & -0.33849 & -0.11616 \\ \mathrm{C} & 3.89140 & 0.53734 & -0.16512 \\ \mathrm{H} & 4.55067 & -0.19204 & 0.30828 \\ \mathrm{H} & 3.94612 & 0.42977 & -1.25132 \\ \mathrm{H} & 4.22851 & 1.53762 & 0.11180 \\ \mathrm{C} & 2.68327 & -2.08392 & -0.23212 \\ \mathrm{H} & 3.45097 & -1.93123 & -0.99115 \\ \mathrm{H} & 3.16131 & -2.34849 & 0.71885 \\ \mathrm{H} & 2.03375 & -2.90181 & -0.53679\end{array}$



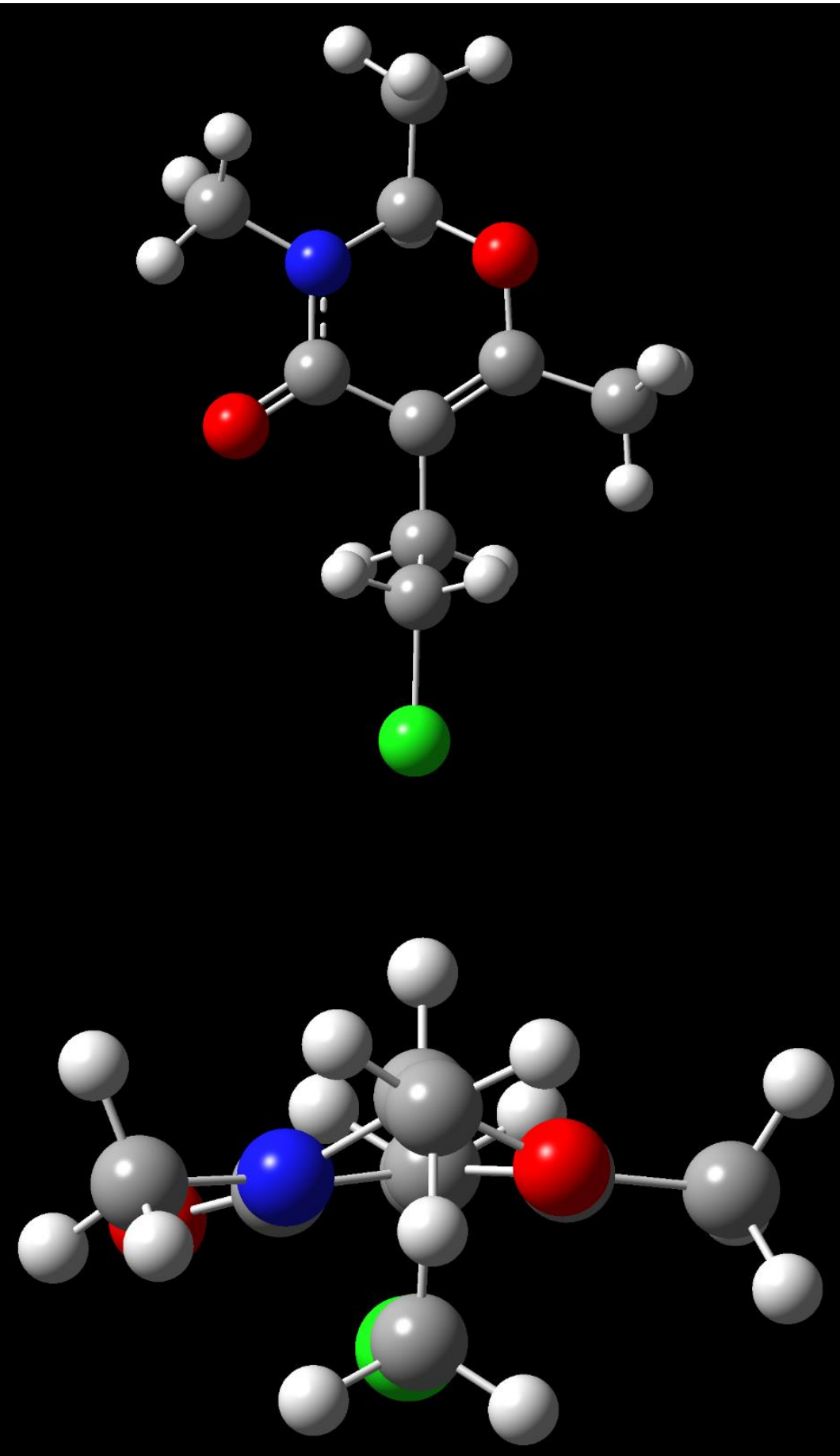


\section{Ketene pathway}

$2 a$ - Acid chloride with chloride

SCF Energy: -1303.647483 a.u.

$\begin{array}{llll}\mathrm{C} & -0.12768 & 1.54675 & -0.09185 \\ \mathrm{C} & 0.49064 & 0.17569 & 0.08522 \\ \mathrm{C} & -0.18957 & -0.89307 & 0.94343 \\ \mathrm{C} & -0.30227 & -0.98255 & -0.52419 \\ \mathrm{H} & -1.06113 & -0.57063 & 1.50171 \\ \mathrm{H} & 0.47309 & -1.57734 & 1.45992 \\ \mathrm{H} & 0.27145 & -1.72959 & -1.05814 \\ \mathrm{H} & -1.25941 & -0.71068 & -0.95998 \\ \mathrm{C} & 1.97519 & 0.16836 & 0.07713 \\ \mathrm{O} & 2.70460 & 1.08922 & 0.24419 \\ \mathrm{O} & 0.43848 & 2.38567 & -0.76444 \\ \mathrm{C} & -1.46357 & 1.80464 & 0.54942 \\ \mathrm{H} & -1.82765 & 2.77732 & 0.21980 \\ \mathrm{H} & -2.19554 & 1.02641 & 0.29853 \\ \mathrm{H} & -1.34589 & 1.80624 & 1.63846 \\ \mathrm{Cl} & 2.73459 & -1.47457 & -0.14127 \\ \mathrm{Cl} & -3.94024 & -0.86307 & -0.16303\end{array}$




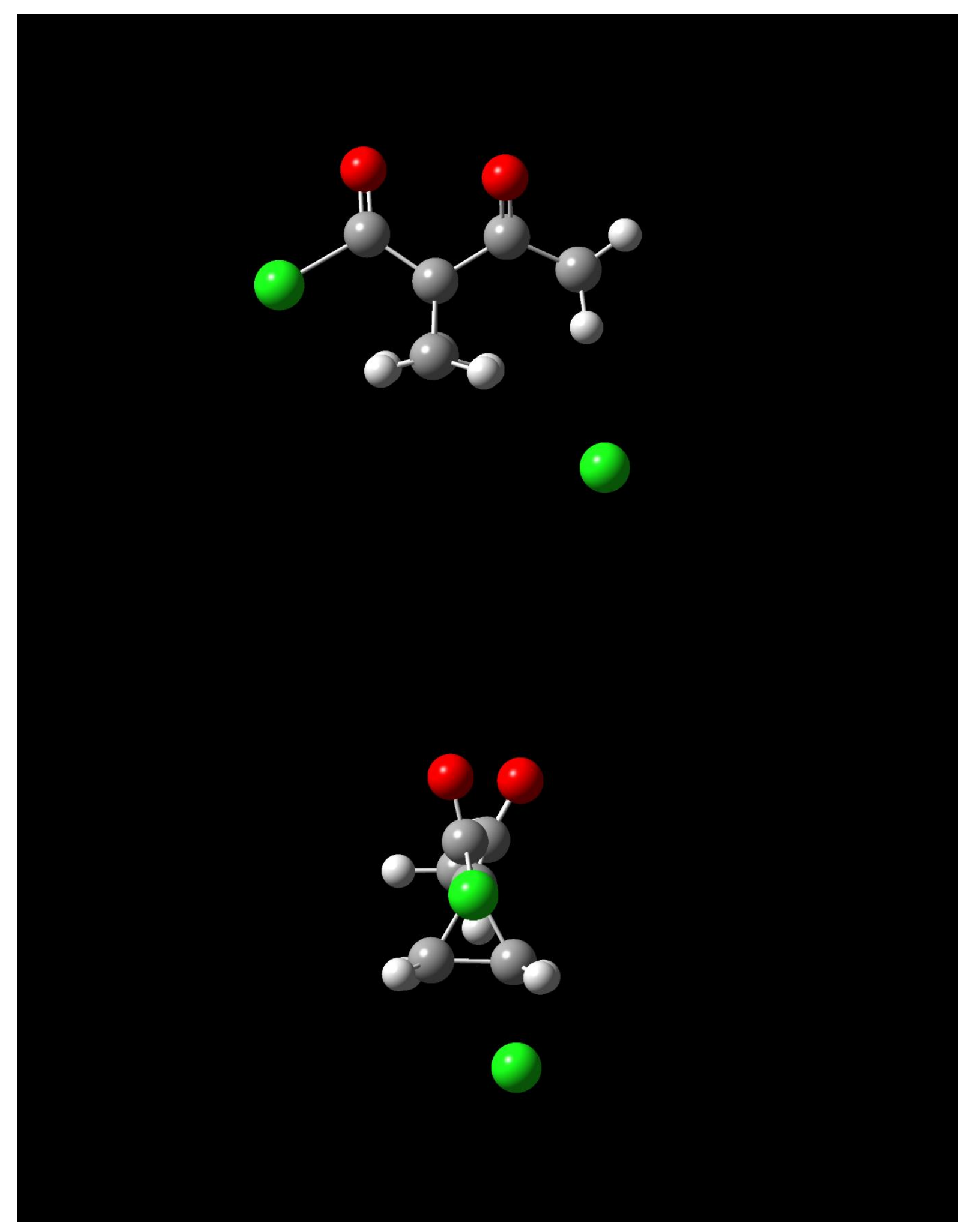


$2 b$ - Acid chloride to enolate transition state

SCF Energy: -1303.612177 a.u.

$\begin{array}{llll}\mathrm{C} & -1.32430 & -1.58804 & -0.00171 \\ \mathrm{C} & -0.70049 & -0.28098 & 0.24192 \\ \mathrm{C} & 0.64385 & -0.19693 & 0.94029 \\ \mathrm{C} & 1.16042 & -0.18412 & -0.42206 \\ \mathrm{H} & 0.88726 & -1.08060 & 1.52361 \\ \mathrm{H} & 0.80245 & 0.71466 & 1.50714 \\ \mathrm{H} & 1.14580 & 0.74246 & -0.97976 \\ \mathrm{H} & 1.22466 & -1.10615 & -0.98384 \\ \mathrm{C} & -1.45607 & 0.87886 & -0.11246 \\ \mathrm{O} & -2.53322 & 1.02005 & -0.60586 \\ \mathrm{O} & -2.41856 & -1.72732 & -0.54148 \\ \mathrm{C} & -0.58015 & -2.82721 & 0.47484 \\ \mathrm{H} & -1.09571 & -3.70266 & 0.08124 \\ \mathrm{H} & 0.46573 & -2.85021 & 0.15755 \\ \mathrm{H} & -0.59359 & -2.86786 & 1.56907 \\ \mathrm{Cl} & -0.56134 & 2.51540 & 0.28209 \\ \mathrm{Cl} & 3.52123 & -0.10369 & -0.30688\end{array}$




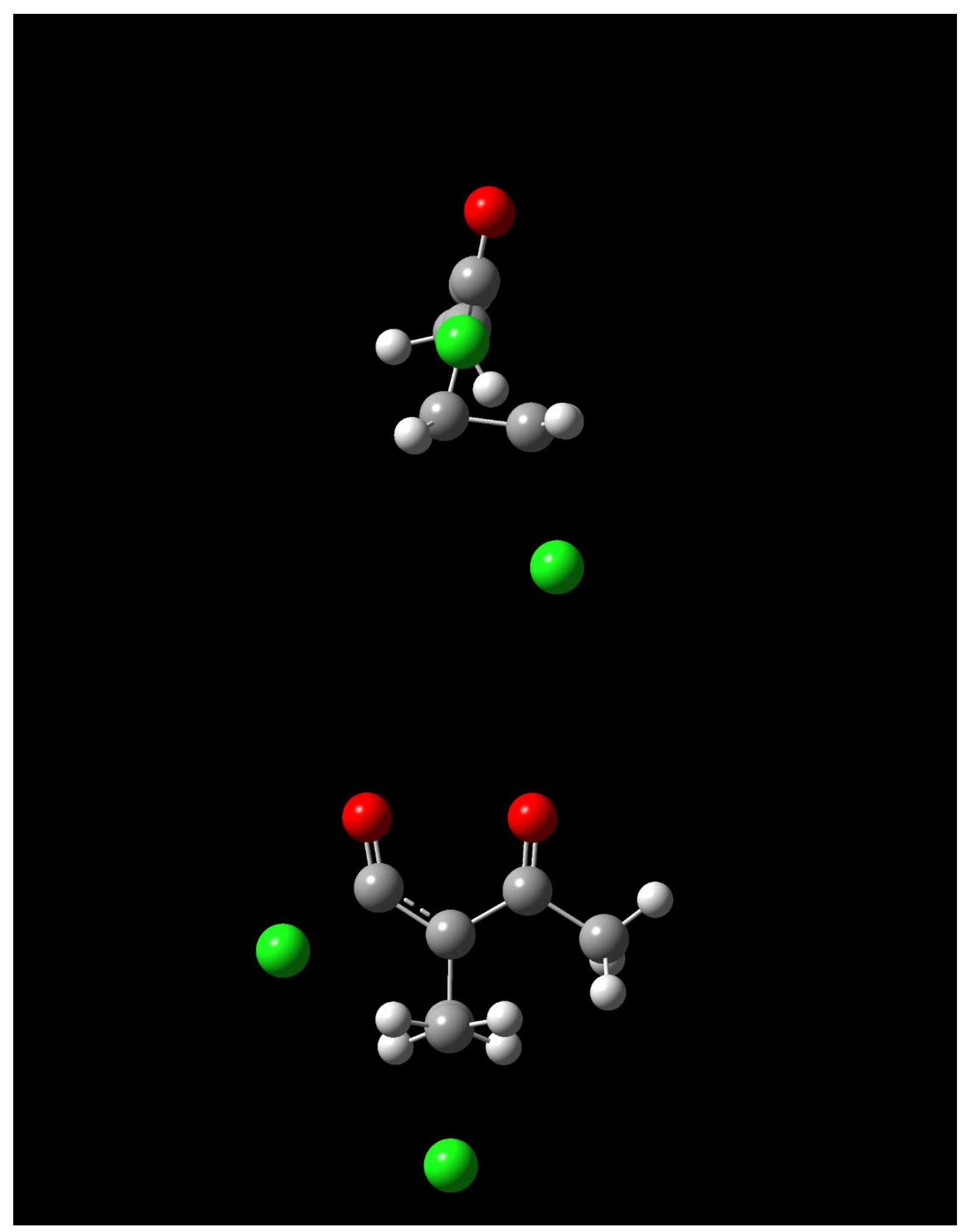


2c-Enolate

SCF Energy: -1303.642669 a.u.

$\begin{array}{llll}\mathrm{C} & -1.53144 & -1.48263 & 0.02702 \\ \mathrm{C} & -0.76610 & -0.26224 & 0.21883 \\ \mathrm{C} & 0.68456 & -0.30412 & 0.62845 \\ \mathrm{C} & 1.59236 & -0.25890 & -0.59288 \\ \mathrm{H} & 0.88972 & -1.21734 & 1.19259 \\ \mathrm{H} & 0.92694 & 0.53478 & 1.28219 \\ \mathrm{H} & 1.47587 & 0.68039 & -1.13283 \\ \mathrm{H} & 1.41563 & -1.09820 & -1.26694 \\ \mathrm{C} & -1.46938 & 0.90484 & -0.04727 \\ \mathrm{O} & -2.55134 & 1.26705 & -0.37025 \\ \mathrm{O} & -2.72036 & -1.52158 & -0.31207 \\ \mathrm{C} & -0.81298 & -2.80466 & 0.27845 \\ \mathrm{H} & -1.46514 & -3.61542 & -0.04653 \\ \mathrm{H} & 0.13944 & -2.86723 & -0.25554 \\ \mathrm{H} & -0.60099 & -2.92536 & 1.34594 \\ \mathrm{Cl} & -0.21198 & 2.57998 & 0.18707 \\ \mathrm{Cl} & 3.34199 & -0.35699 & -0.11271\end{array}$




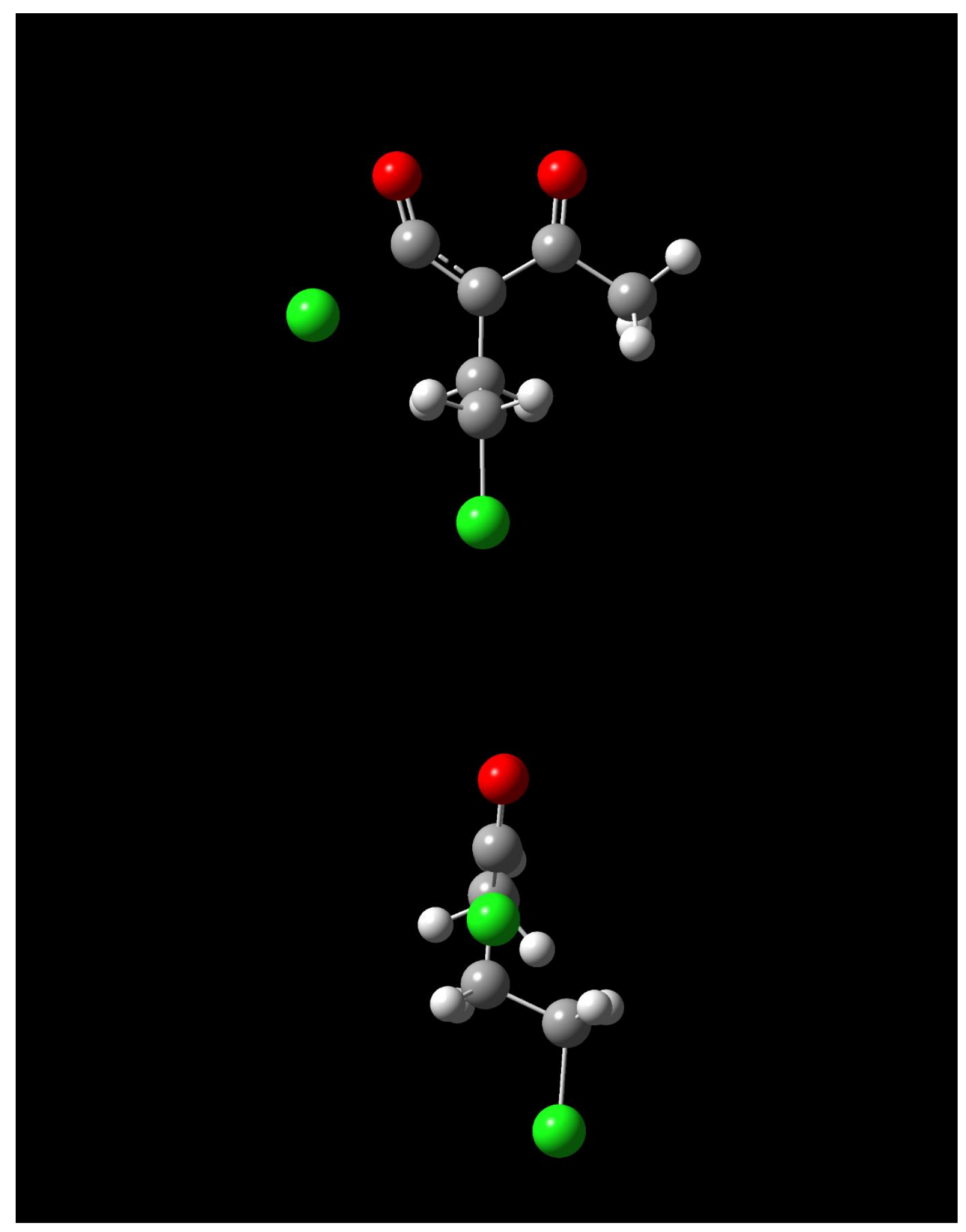


$2 d$-Enolate to ketene transition state

SCF Energy: -1303.642006 a.u.

$\begin{array}{llll}\mathrm{C} & -1.737973 & -1.34663 & 0.01441 \\ \mathrm{C} & -0.83303 & -0.21476 & 0.22750 \\ \mathrm{C} & 0.60606 & -0.39821 & 0.63295 \\ \mathrm{C} & 1.50728 & -0.46006 & -0.59192 \\ \mathrm{H} & 0.70969 & -1.32073 & 1.21003 \\ \mathrm{H} & 0.92621 & 0.42704 & 1.26861 \\ \mathrm{H} & 1.48762 & 0.48806 & -1.12808 \\ \mathrm{H} & 1.23956 & -1.277901 & -1.26241 \\ \mathrm{C} & -1.45086 & 0.97750 & -0.02240 \\ \mathrm{O} & -2.40106 & 1.59678 & -0.29466 \\ \mathrm{O} & -2.91524 & -1.23080 & -0.32967 \\ \mathrm{C} & -1.17021 & -2.73655 & 0.25287 \\ \mathrm{H} & -1.90222 & -3.46835 & -0.08807 \\ \mathrm{H} & -0.22456 & -2.88984 & -0.27385 \\ \mathrm{H} & -0.98291 & -2.88747 & 1.32086 \\ \mathrm{Cl} & 0.27587 & 2.67781 & 0.15326 \\ \mathrm{Cl} & 3.23165 & -0.74493 & -0.10873\end{array}$




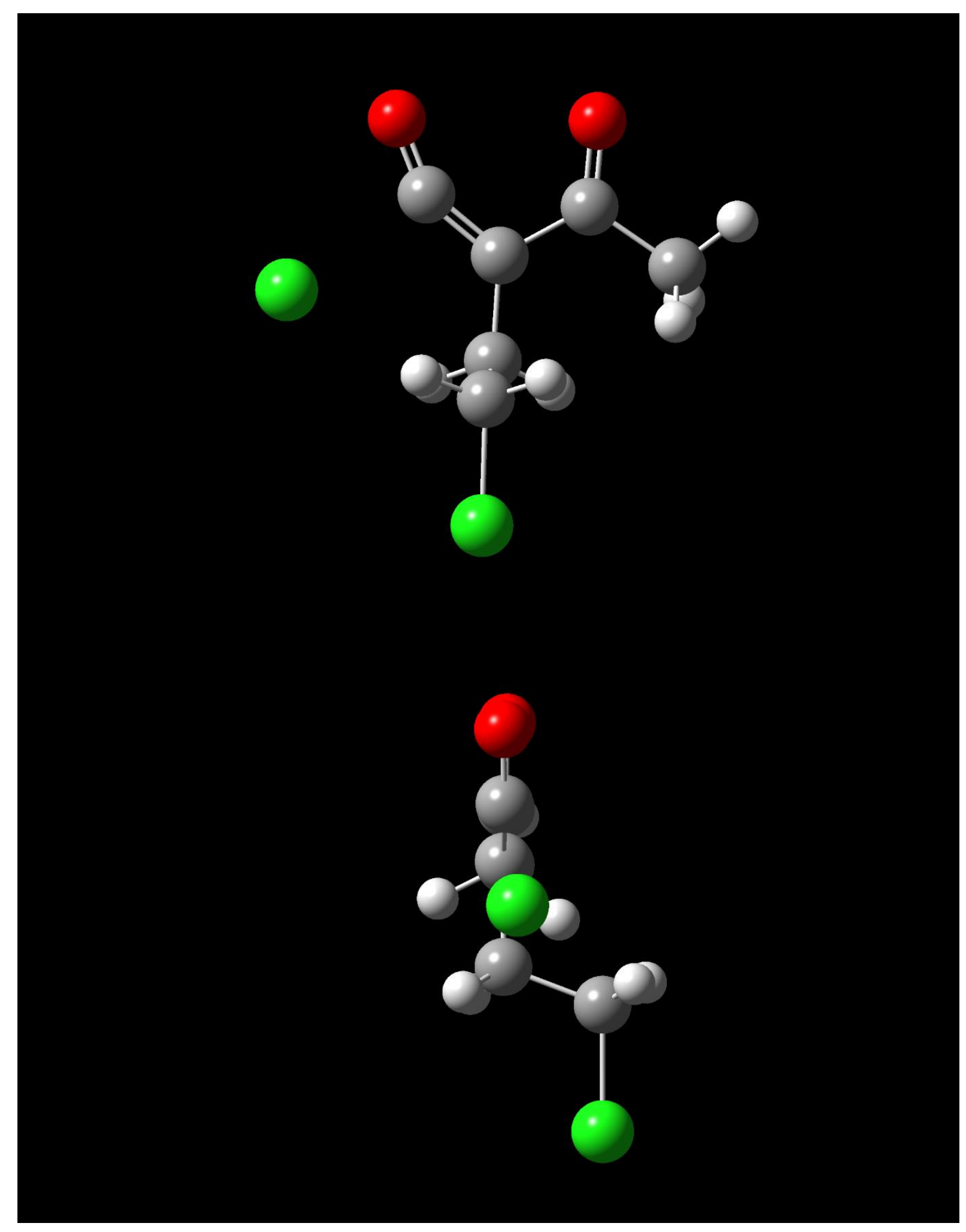


2e1 - Ketene plus chloride 10 Angstrom away

SCF Energy: -1303.638573 a.u.

$\begin{array}{llll}\mathrm{C} & 3.55233 & -0.92031 & 0.12621 \\ \mathrm{C} & 2.08794 & -0.76832 & 0.19277 \\ \mathrm{C} & 1.35099 & 0.50118 & 0.57099 \\ \mathrm{C} & 1.14145 & 1.39209 & -0.64847 \\ \mathrm{H} & 1.93146 & 1.03463 & 1.32881 \\ \mathrm{H} & 0.38358 & 0.26676 & 1.02180 \\ \mathrm{H} & 0.47156 & 0.92634 & -1.37119 \\ \mathrm{H} & 2.08247 & 1.64204 & -1.13936 \\ \mathrm{C} & 1.41607 & -1.87688 & -0.12902 \\ \mathrm{O} & 0.84684 & -2.84433 & -0.39524 \\ \mathrm{O} & 4.07195 & -1.96692 & -0.24391 \\ \mathrm{C} & 4.38373 & 0.25981 & 0.56785 \\ \mathrm{H} & 5.42988 & 0.05455 & 0.34377 \\ \mathrm{H} & 4.07279 & 1.18266 & 0.07094 \\ \mathrm{H} & 4.26691 & 0.40905 & 1.64628 \\ \mathrm{Cl} & -8.70879 & -0.51465 & 0.11194 \\ \mathrm{Cl} & 0.38032 & 2.95281 & -0.16311\end{array}$




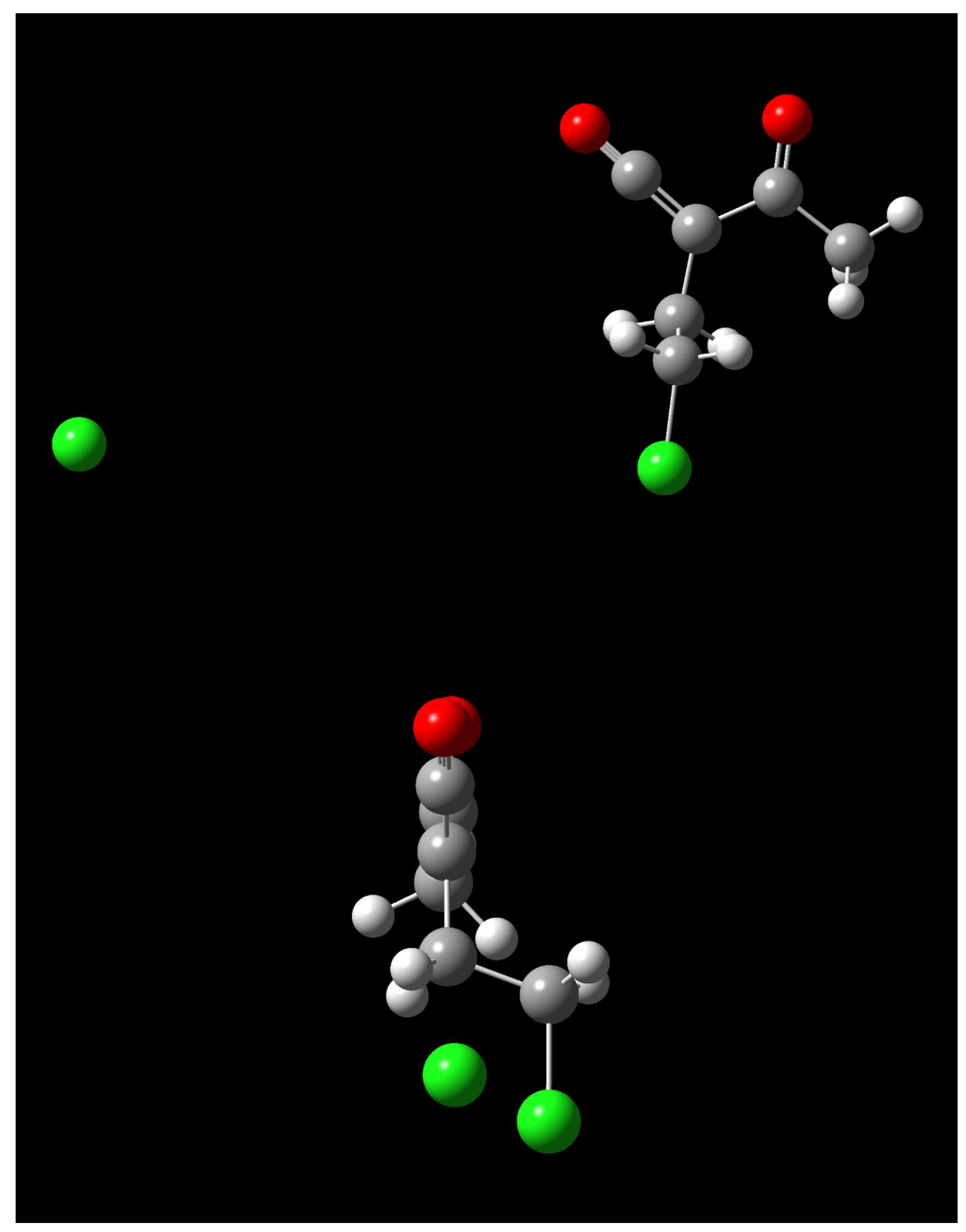


$2 e 2$ - Ketene plus imine

SCF Energy: -1016.497686 a.u.

$\begin{array}{llll}\mathrm{C} & -0.33756 & 1.63443 & -0.44426 \\ \mathrm{C} & 0.32291 & 0.42444 & -0.95441 \\ \mathrm{C} & 1.81099 & 0.13310 & -0.91166 \\ \mathrm{C} & 2.20676 & -0.57119 & 0.38093 \\ \mathrm{H} & 2.35943 & 1.07456 & -1.00685 \\ \mathrm{H} & 2.10212 & -0.48723 & -1.76334 \\ \mathrm{H} & 1.70427 & -1.53361 & 0.48228 \\ \mathrm{H} & 2.00044 & 0.03791 & 1.26138 \\ \mathrm{C} & -0.51514 & -0.46496 & -1.49703 \\ \mathrm{O} & -1.22640 & -1.22414 & -1.99439 \\ \mathrm{O} & -1.54827 & 1.79924 & -0.55585 \\ \mathrm{C} & 0.54171 & 2.68078 & 0.19806 \\ \mathrm{H} & -0.09349 & 3.42546 & 0.67690 \\ \mathrm{H} & 1.22339 & 2.24652 & 0.93464 \\ \mathrm{H} & 1.15141 & 3.16855 & -0.56979 \\ \mathrm{Cl} & 3.97979 & -0.89949 & 0.39658 \\ \mathrm{C} & -2.99464 & -0.48518 & 0.77586 \\ \mathrm{H} & -3.40592 & 0.50104 & 1.03804 \\ \mathrm{~N} & -1.90514 & -0.88967 & 1.28712 \\ \mathrm{C} & -1.25597 & -0.00243 & 2.23688 \\ \mathrm{H} & -1.19698 & -0.49685 & 3.21104 \\ \mathrm{H} & -0.22659 & 0.18136 & 1.91133 \\ \mathrm{H} & -1.76913 & 0.96302 & 2.35047 \\ \mathrm{C} & -3.78034 & -1.29731 & -0.20366 \\ \mathrm{H} & -3.29072 & -2.25333 & -0.39469 \\ \mathrm{H} & -4.79081 & -1.47100 & 0.18037 \\ \mathrm{H} & -3.88296 & -0.74810 & -1.14573\end{array}$



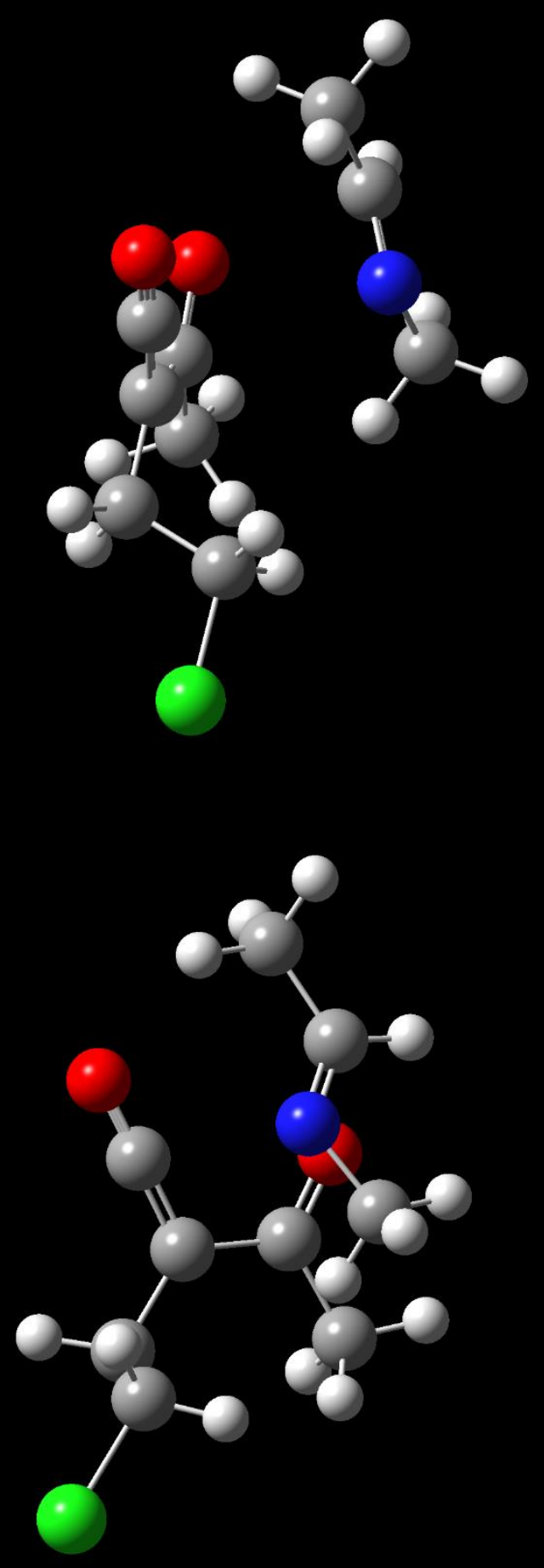
$2 f$ - Ketene and imine to iminium transition state

SCF Energy: -1016.489761 a.u.

$\begin{array}{llll}\mathrm{C} & -0.22605 & 1.68065 & -0.36454 \\ \mathrm{C} & 0.27172 & 0.34971 & -0.68999 \\ \mathrm{C} & 1.74991 & 0.02229 & -0.85483 \\ \mathrm{C} & 2.32442 & -0.53262 & 0.44286 \\ \mathrm{H} & 2.29155 & 0.92573 & -1.14674 \\ \mathrm{H} & 1.90005 & -0.70665 & -1.65692 \\ \mathrm{H} & 1.86529 & -1.48677 & 0.70480 \\ \mathrm{H} & 2.20341 & 0.16396 & 1.27337 \\ \mathrm{C} & -0.55387 & -0.70449 & -0.89011 \\ \mathrm{O} & -0.89875 & -1.73728 & -1.30198 \\ \mathrm{O} & -1.41179 & 1.99378 & -0.43891 \\ \mathrm{C} & 0.80454 & 2.72199 & 0.02899 \\ \mathrm{H} & 0.28569 & 3.56904 & 0.47769 \\ \mathrm{H} & 1.55083 & 2.33201 & 0.72582 \\ \mathrm{H} & 1.33372 & 3.07218 & -0.86382 \\ \mathrm{Cl} & 4.09745 & -0.84181 & 0.28178 \\ \mathrm{C} & -3.35258 & -0.63026 & 0.66458 \\ \mathrm{H} & -3.97490 & -0.66081 & 1.57056 \\ \mathrm{~N} & -2.10506 & -0.43123 & 0.75614 \\ \mathrm{C} & -1.49939 & -0.23122 & 2.05712 \\ \mathrm{H} & -0.73034 & -0.99322 & 2.21682 \\ \mathrm{H} & -1.00596 & 0.74602 & 2.07061 \\ \mathrm{H} & -2.22996 & -0.27569 & 2.87401 \\ \mathrm{C} & -4.05121 & -0.81419 & -0.64409 \\ \mathrm{H} & -3.35858 & -0.71294 & -1.47962 \\ \mathrm{H} & -4.52723 & -1.79912 & -0.68184 \\ \mathrm{H} & -4.84542 & -0.06767 & -0.74081\end{array}$




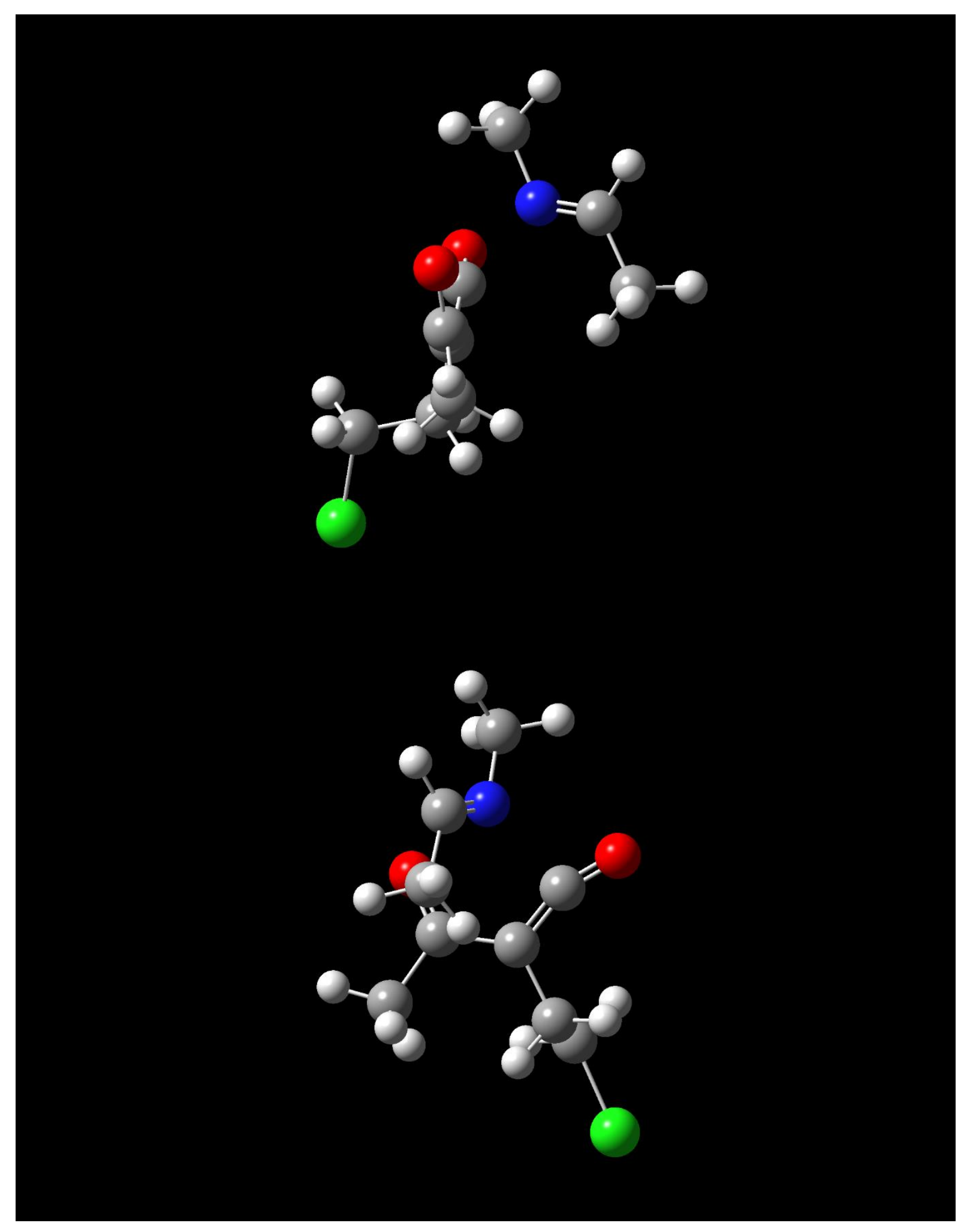


$2 g$ - Ketene pathway iminium intermediate

SCF Energy: -1016.521036 a.u.

$\begin{array}{llll}\mathrm{C} & -0.41212 & 1.65916 & 0.03545 \\ \mathrm{C} & 0.07337 & 0.32038 & -0.21027 \\ \mathrm{C} & 1.48431 & 0.05390 & -0.68544 \\ \mathrm{C} & 2.38038 & -0.27984 & 0.49929 \\ \mathrm{H} & 1.89469 & 0.91065 & -1.22478 \\ \mathrm{H} & 1.48675 & -0.79701 & -1.37408 \\ \mathrm{H} & 2.02521 & -1.16979 & 1.01925 \\ \mathrm{H} & 2.45644 & 0.55158 & 1.20116 \\ \mathrm{C} & -0.68449 & -0.83186 & 0.00239 \\ \mathrm{O} & -0.37831 & -2.01806 & -0.13742 \\ \mathrm{O} & -1.55191 & 1.93045 & 0.45061 \\ \mathrm{C} & 0.53992 & 2.81309 & -0.24004 \\ \mathrm{H} & 0.07269 & 3.73616 & 0.10287 \\ \mathrm{H} & 1.50035 & 2.68097 & 0.26698 \\ \mathrm{H} & 0.74429 & 2.89309 & -1.31279 \\ \mathrm{Cl} & 4.07546 & -0.64423 & -0.03586 \\ \mathrm{C} & -3.09037 & -0.61049 & -0.31460 \\ \mathrm{H} & -4.08251 & -0.54111 & 0.12911 \\ \mathrm{~N} & -2.10311 & -0.64291 & 0.49278 \\ \mathrm{C} & -2.24346 & -0.58501 & 1.94789 \\ \mathrm{H} & -1.75540 & -1.46452 & 2.37054 \\ \mathrm{H} & -1.75467 & 0.32872 & 2.28870 \\ \mathrm{H} & -3.29981 & -0.57047 & 2.21370 \\ \mathrm{C} & -2.96607 & -0.62918 & -1.79054 \\ \mathrm{H} & -1.94172 & -0.78203 & -2.12699 \\ \mathrm{H} & -3.61477 & -1.41158 & -2.19298 \\ \mathrm{H} & -3.33953 & 0.32771 & -2.17086\end{array}$




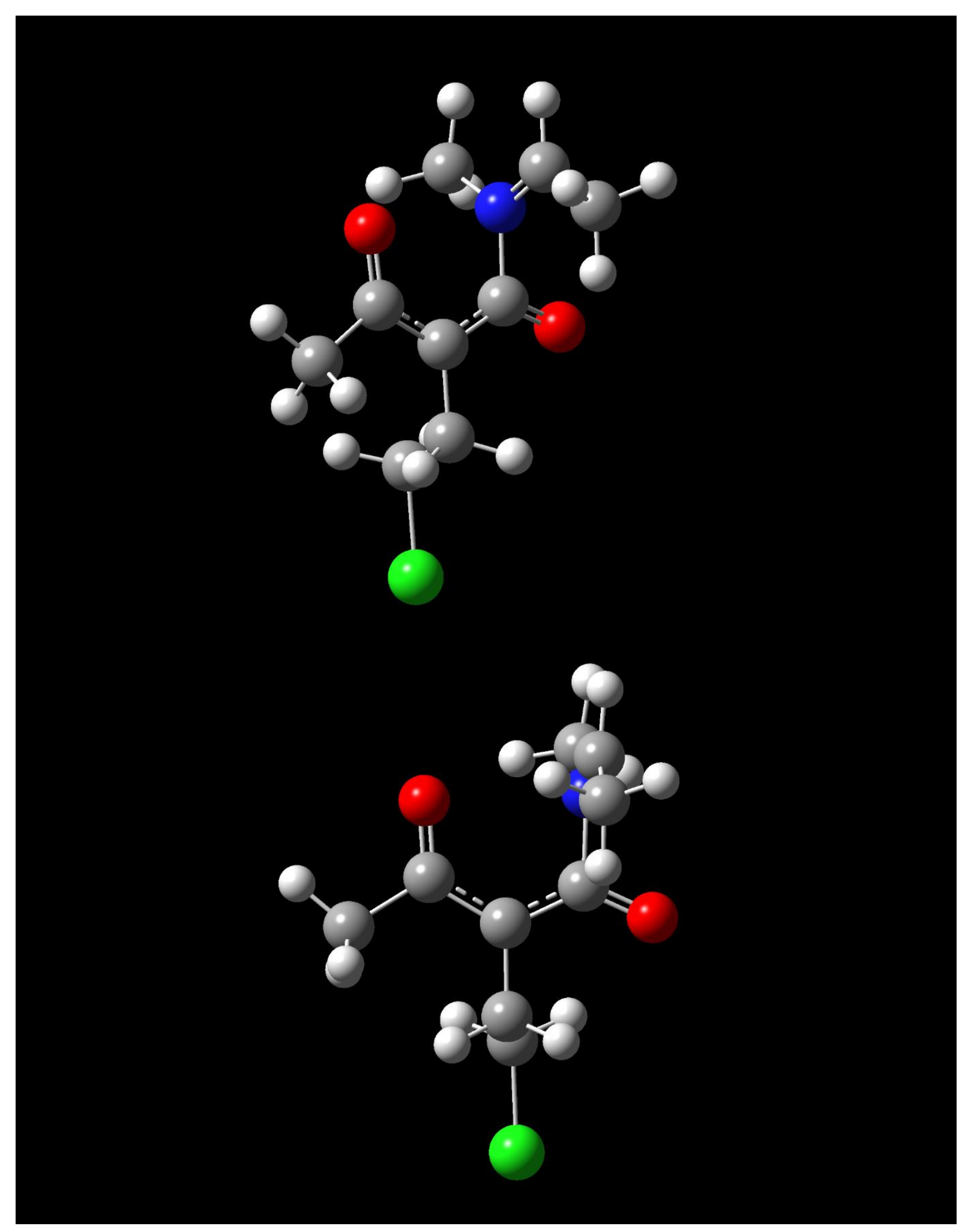


$2 h$ - Ketene pathway iminium intermediate to oxazinone transition state

SCF Energy: -1016.516499 a.u.

\begin{tabular}{|c|c|c|c|}
\hline $\mathrm{C}$ & $0-0.60615$ & 1.46749 & 0.25644 \\
\hline $\mathrm{C}$ & 00.01293 & 0.28676 & -0.27275 \\
\hline C & 01.46549 & 0.28449 & -0.69565 \\
\hline C & 02.34243 & -0.26741 & 0.42028 \\
\hline $\mathrm{H}$ & 01.80689 & 1.28402 & -0.97464 \\
\hline $\mathrm{H}$ & 01.58287 & -0.35533 & -1.57599 \\
\hline $\mathrm{H}$ & 02.05687 & -1.28917 & 0.67060 \\
\hline $\mathrm{H}$ & 02.30808 & 0.35503 & 1.31516 \\
\hline $\mathrm{C}$ & $0-0.60671$ & -0.98011 & -0.31483 \\
\hline 0 & $0-0.13154$ & -2.05972 & -0.66107 \\
\hline 0 & $0-1.83297$ & 1.62085 & 0.49394 \\
\hline $\mathrm{C}$ & 00.27599 & 2.67427 & 0.52477 \\
\hline $\mathrm{H}$ & $0-0.26787$ & 3.36510 & 1.16897 \\
\hline $\mathrm{H}$ & 01.22813 & 2.40854 & 0.98879 \\
\hline $\mathrm{H}$ & 00.49556 & 3.18268 & -0.42057 \\
\hline $\mathrm{Cl}$ & 04.08434 & -0.33166 & -0.08063 \\
\hline $\mathrm{C}$ & $0-2.88510$ & -0.20108 & -0.14455 \\
\hline $\mathrm{H}$ & $0-3.76239$ & -0.10530 & 0.49242 \\
\hline $\mathrm{N}$ & $0-1.96601$ & -1.01116 & 0.27743 \\
\hline $\mathrm{C}$ & $0-2.02689$ & -1.63797 & 1.59988 \\
\hline $\mathrm{H}$ & $0-1.61504$ & -2.64368 & 1.52076 \\
\hline $\mathrm{H}$ & $0-1.43282$ & -1.04948 & 2.30519 \\
\hline $\mathrm{H}$ & $0-3.06344$ & -1.68615 & 1.93079 \\
\hline $\mathrm{C}$ & $0-2.99529$ & 0.29667 & -1.53989 \\
\hline $\mathrm{H}$ & $0-2.03477$ & 0.32263 & -2.05252 \\
\hline $\mathrm{H}$ & $0-3.66897$ & -0.38727 & -2.06927 \\
\hline $\mathrm{H}$ & $0-3.44899$ & 1.28707 & -1.53614 \\
\hline
\end{tabular}




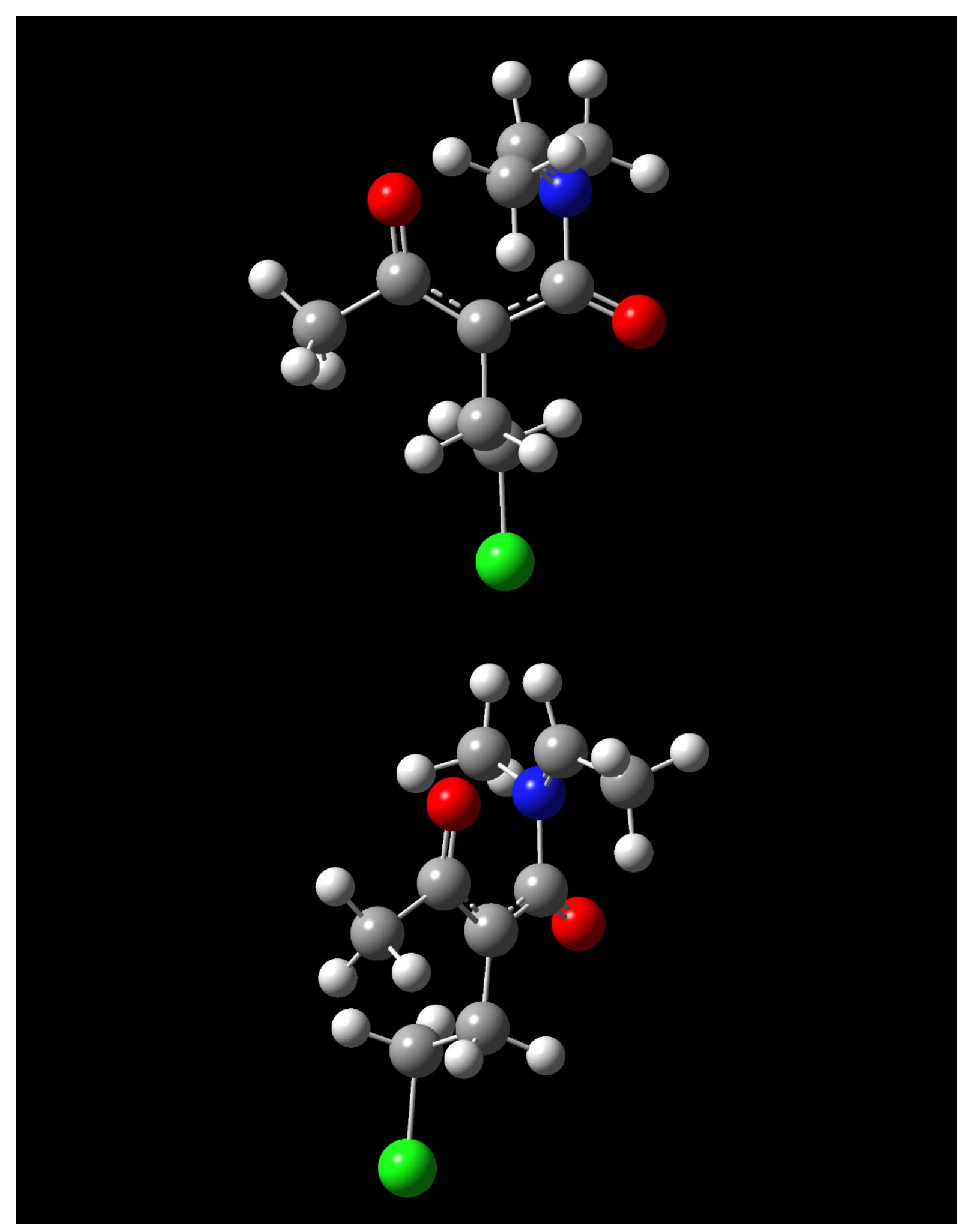


$2 i$ - Oxazinone from ketene

SCF Energy: -1016.563421 a.u.

$\begin{array}{llll}\mathrm{C} & 0.45740 & 1.40165 & -0.21913 \\ \mathrm{C} & -0.12188 & 0.25763 & 0.20490 \\ \mathrm{C} & -1.54568 & 0.15865 & 0.68544 \\ \mathrm{C} & -2.46975 & -0.19191 & -0.47318 \\ \mathrm{H} & -1.87034 & 1.09145 & 1.15264 \\ \mathrm{H} & -1.61096 & -0.63041 & 1.43945 \\ \mathrm{H} & -2.18663 & -1.14239 & -0.92451 \\ \mathrm{H} & -2.48234 & 0.59124 & -1.23232 \\ \mathrm{C} & 0.61376 & -1.01654 & 0.03969 \\ \mathrm{O} & 0.06348 & -2.11237 & 0.17543 \\ \mathrm{O} & 1.75174 & 1.43521 & -0.63009 \\ \mathrm{C} & -0.18060 & 2.74538 & -0.34419 \\ \mathrm{H} & 0.01369 & 3.13769 & -1.34644 \\ \mathrm{H} & -1.25665 & 2.70659 & -0.18211 \\ \mathrm{H} & 0.26880 & 3.43742 & 0.37469 \\ \mathrm{Cl} & -4.17732 & -0.37671 & 0.09576 \\ \mathrm{C} & 2.58803 & 0.37719 & -0.16732 \\ \mathrm{H} & 3.45517 & 0.39205 & -0.83089 \\ \mathrm{~N} & 1.91569 & -0.89705 & -0.35374 \\ \mathrm{C} & 2.73293 & -2.08764 & -0.51927 \\ \mathrm{H} & 2.12068 & -2.87595 & -0.95516 \\ \mathrm{H} & 3.56169 & -1.85624 & -1.19131 \\ \mathrm{H} & 3.13324 & -2.44403 & 0.43707 \\ \mathrm{C} & 3.01727 & 0.62823 & 1.27172 \\ \mathrm{H} & 2.15027 & 0.64997 & 1.93703 \\ \mathrm{H} & 3.68762 & -0.17189 & 1.59618 \\ \mathrm{H} & 3.54982 & 1.57934 & 1.33912 \\ & & & \\ & & & \end{array}$



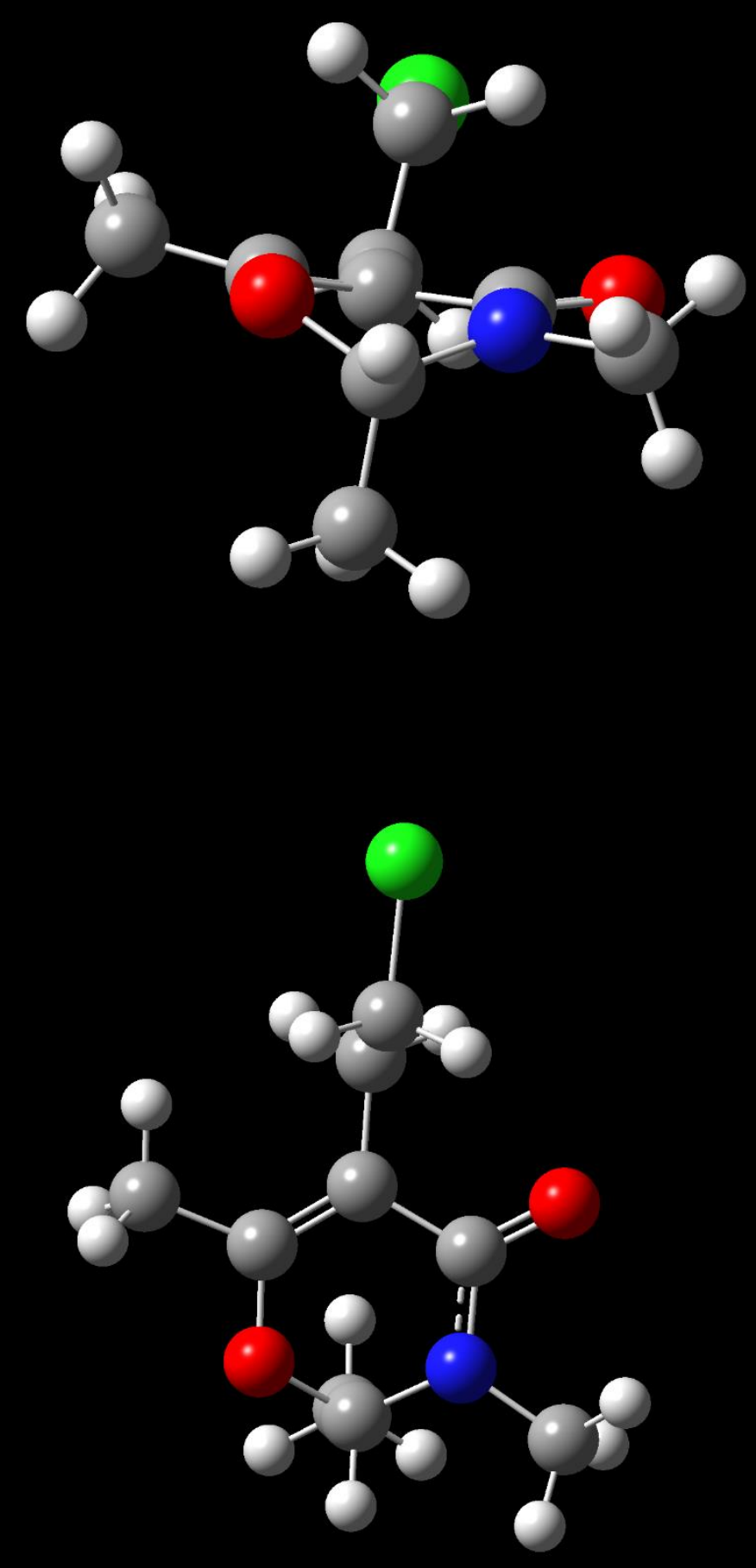


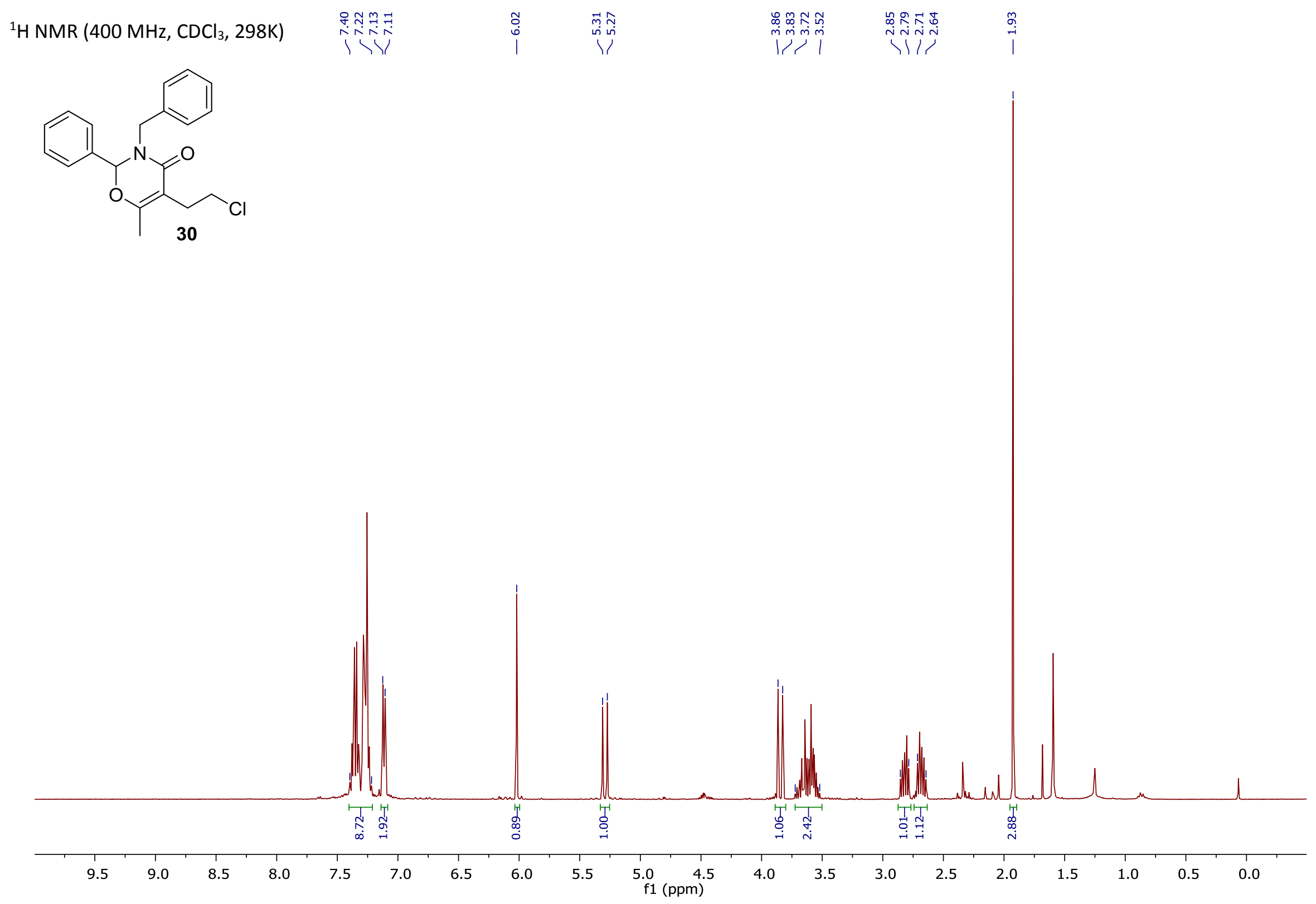


$\left.{ }^{1} \mathrm{H} \mathrm{NMR} \mathrm{(400} \mathrm{MHz,} \mathrm{CDCl}_{3}, 298 \mathrm{~K}\right)$
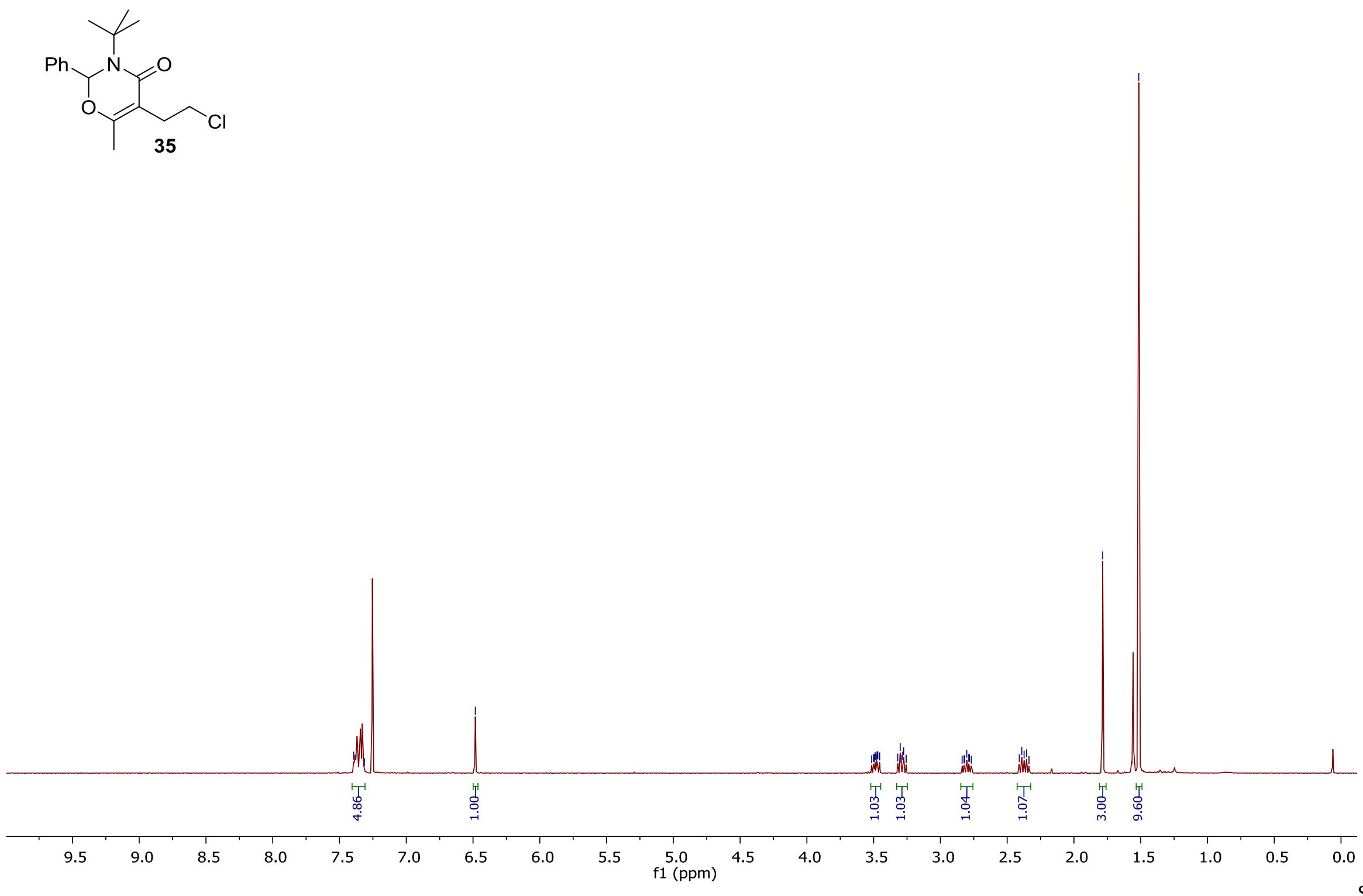
${ }^{13} \mathrm{C}$ NMR (100 MHz, $\left.\mathrm{CDCl}_{3}, 298 \mathrm{~K}\right)$

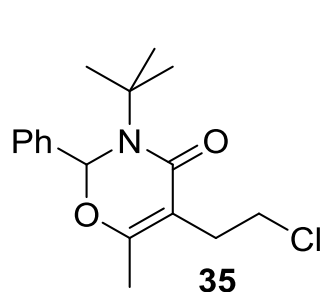

ind

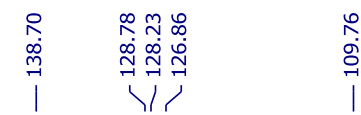

$\underset{\substack{m \\ \infty}}{\substack{\infty \\ \mid}}$

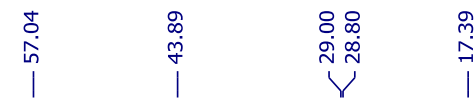

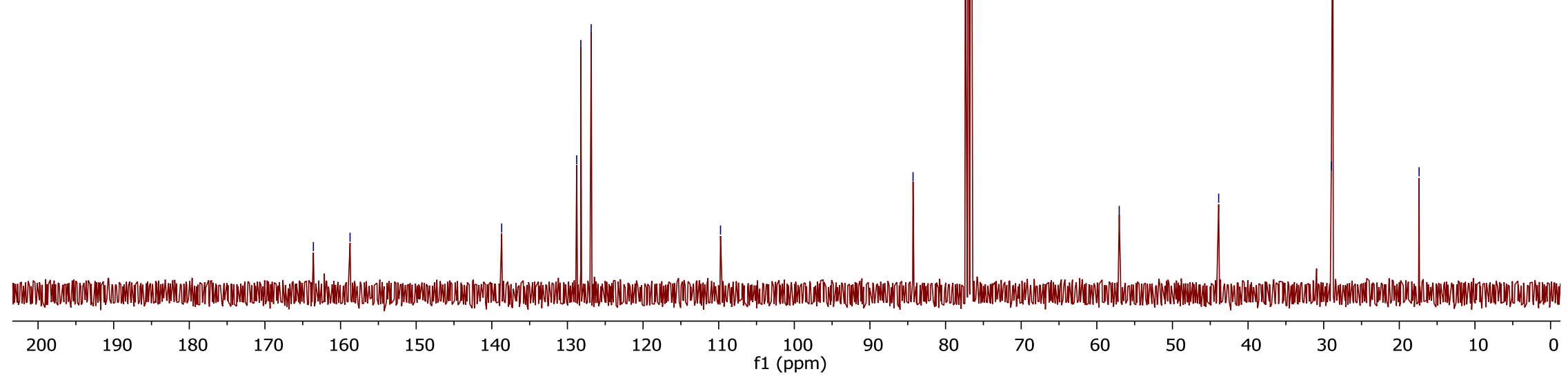




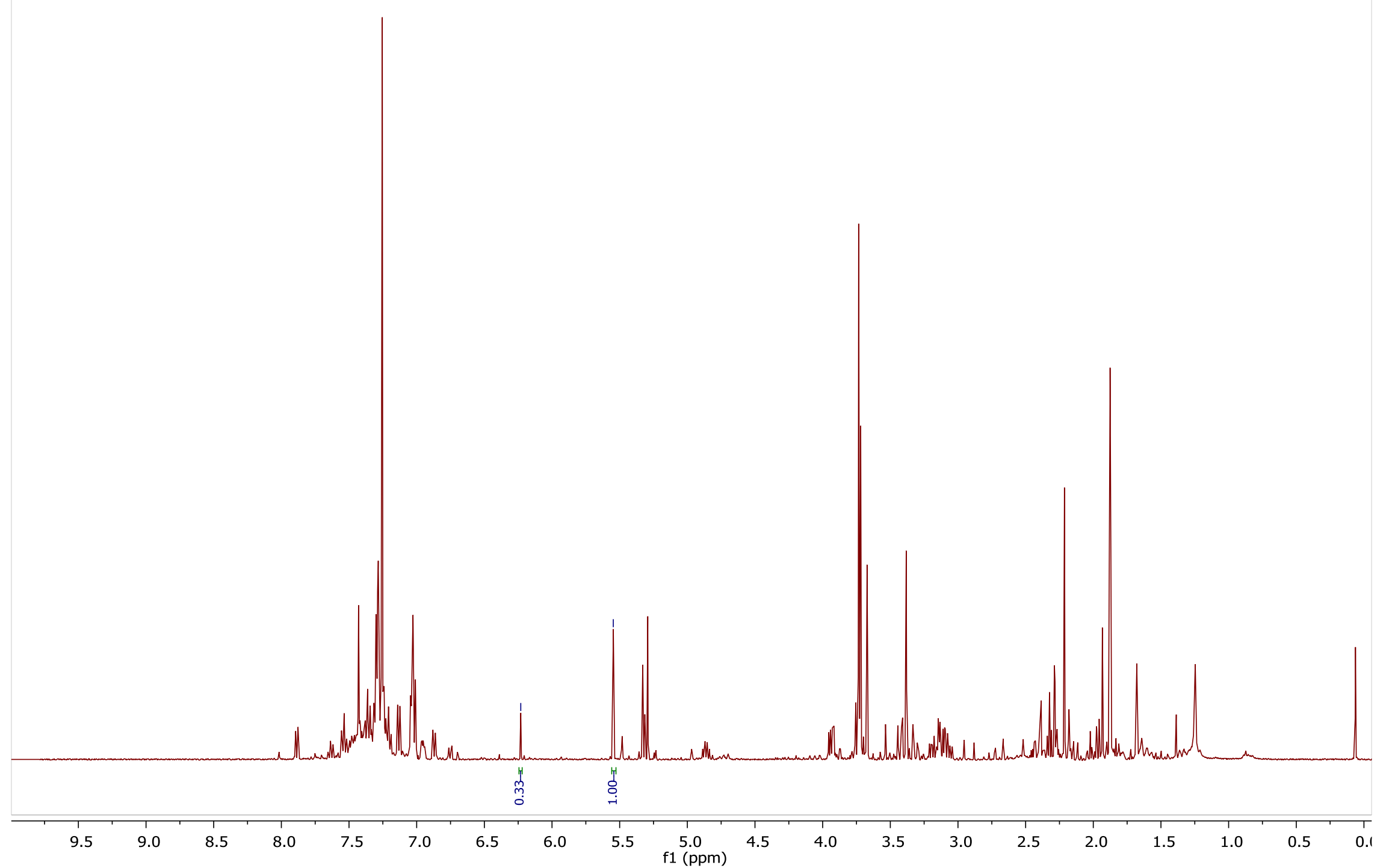


${ }^{1} \mathrm{H} \mathrm{NMR}\left(400 \mathrm{MHz}, \mathrm{CDCl}_{3}, 298 \mathrm{~K}\right)$

m m m లై

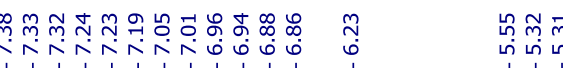
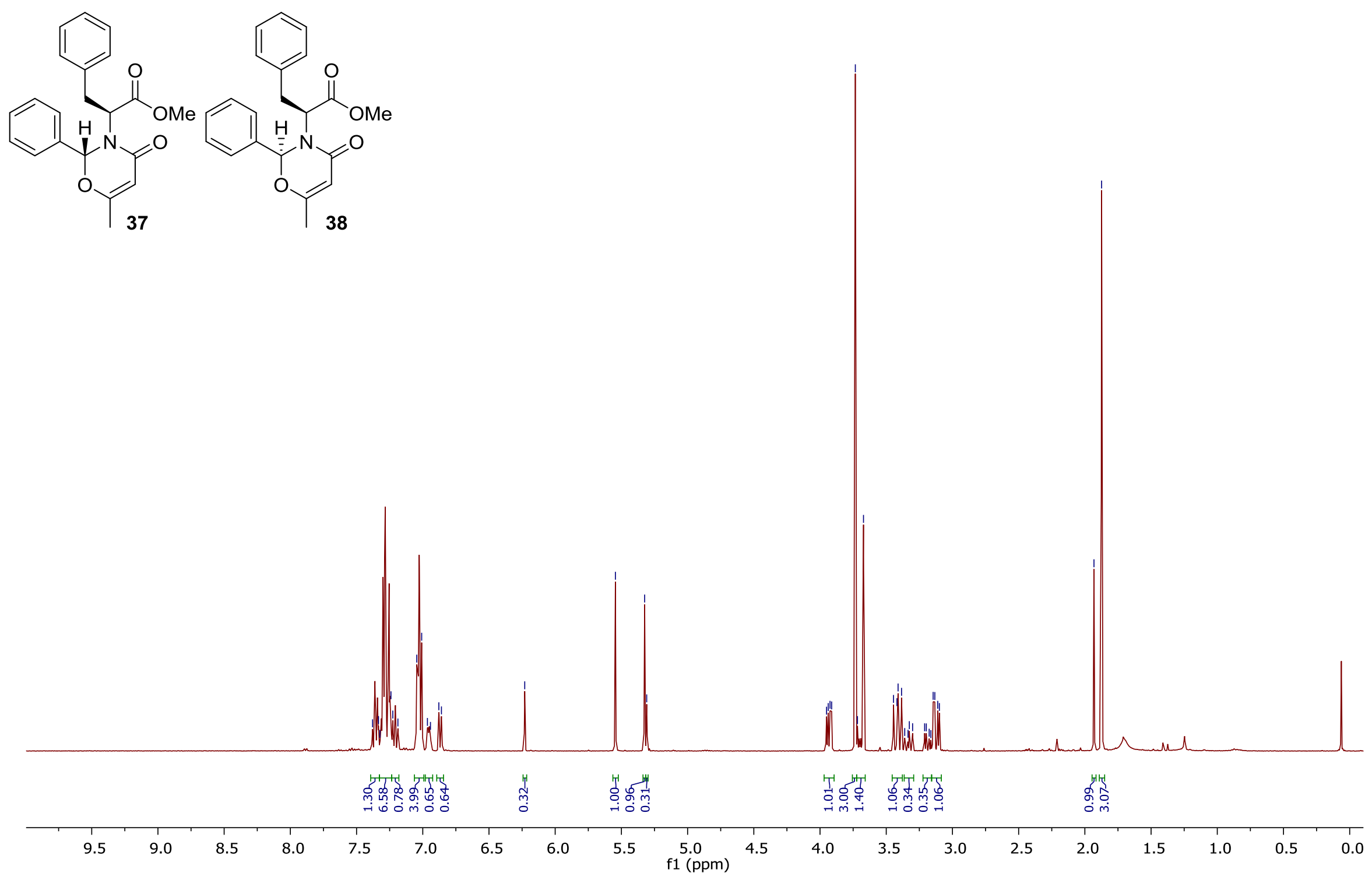


$$
\text { It }
$$




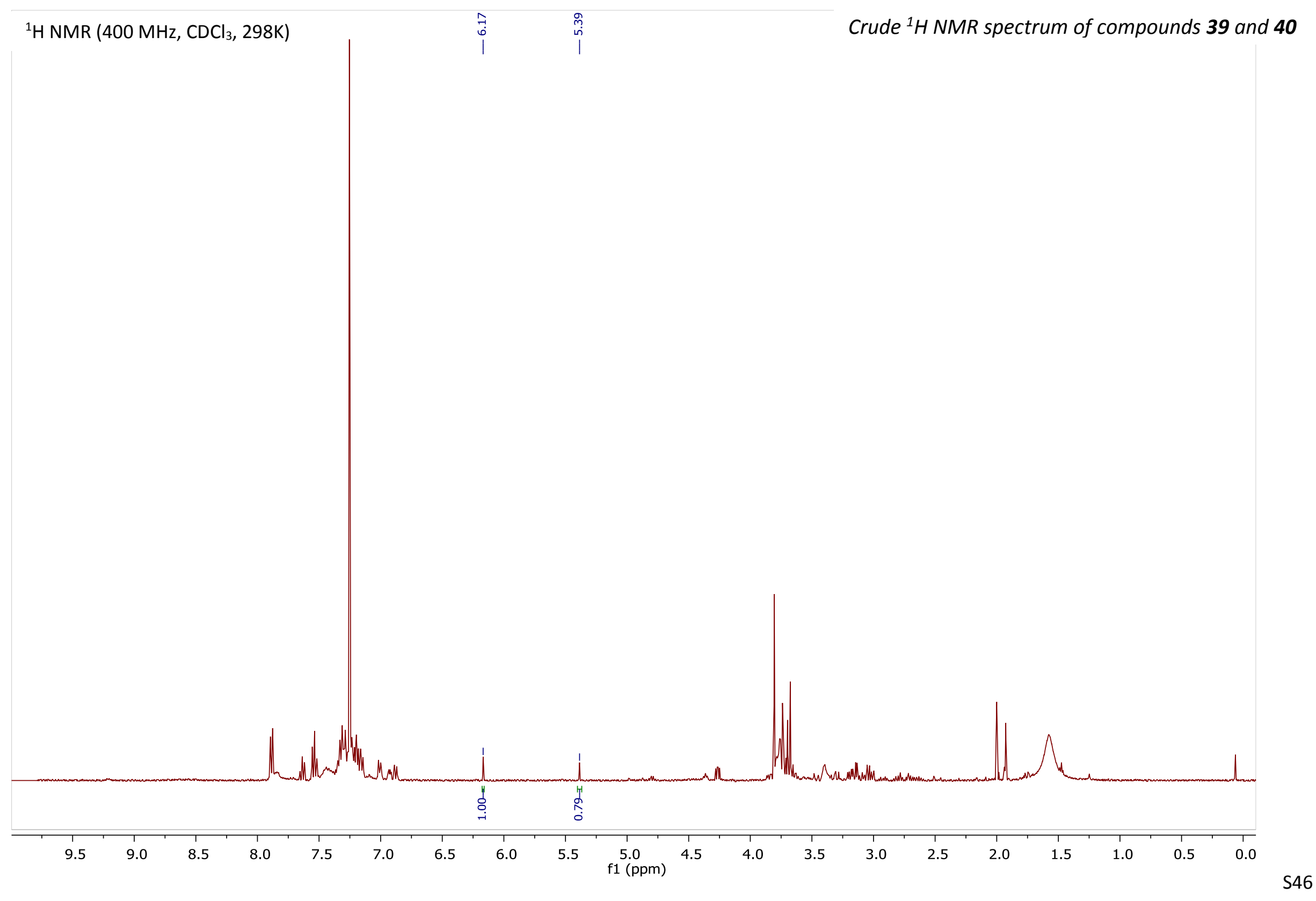




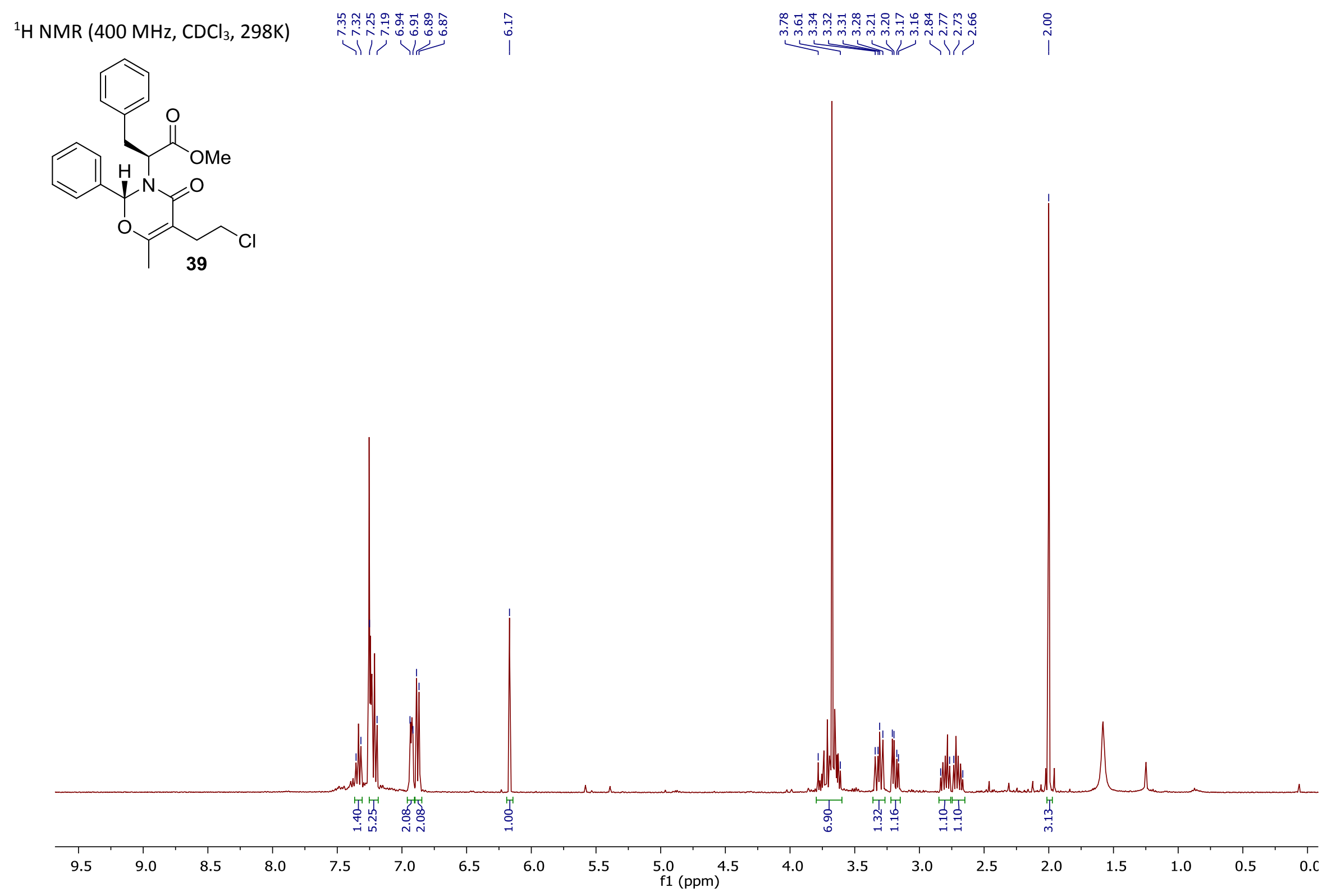


${ }^{13} \mathrm{C}$ NMR (100 MHz, $\left.\mathrm{CDCl}_{3}, 298 \mathrm{~K}\right)$

|
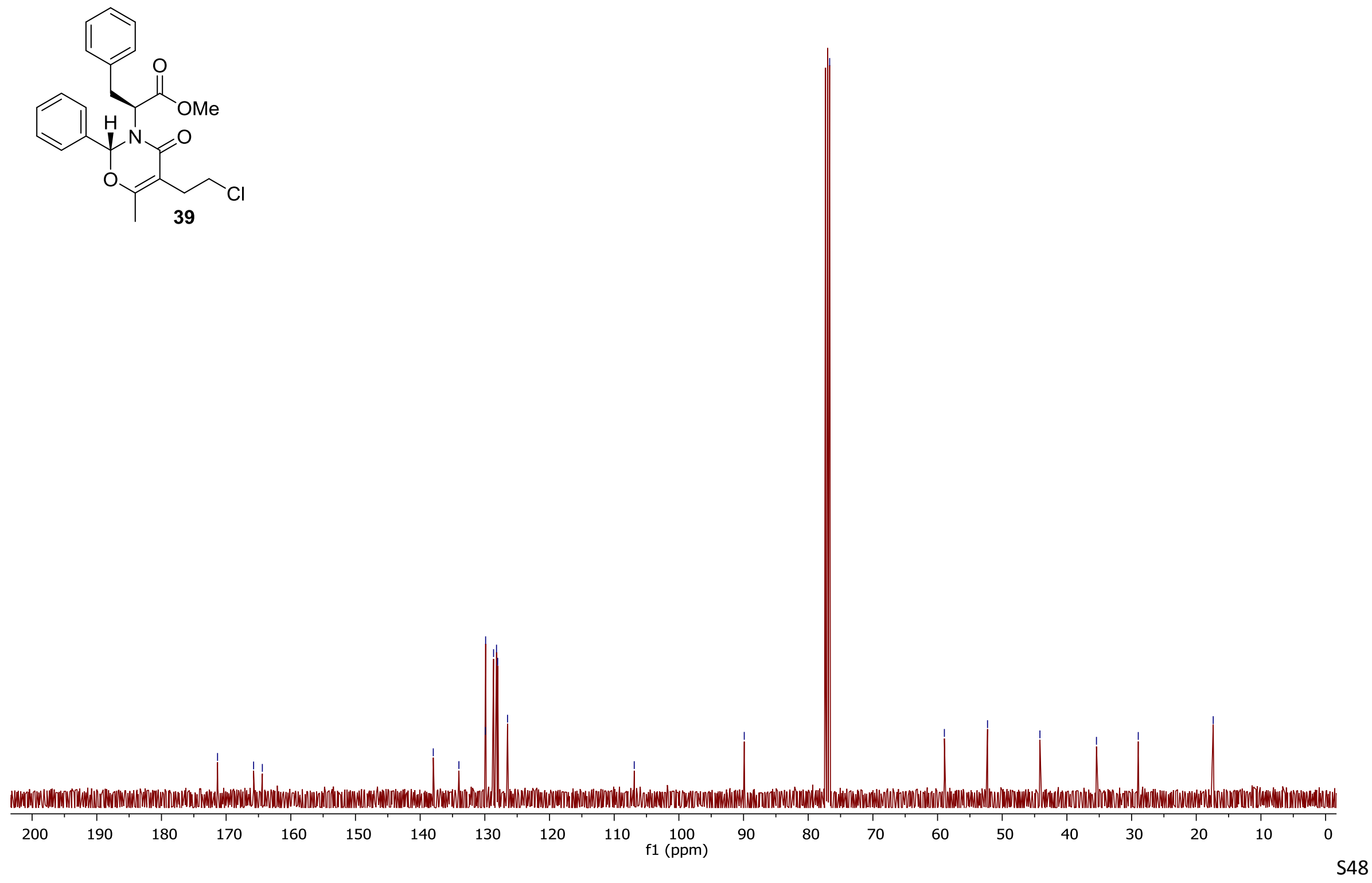
${ }^{1} \mathrm{H}$ NMR (400 MHz, $\left.\mathrm{CDCl}_{3}, 298 \mathrm{~K}\right)$

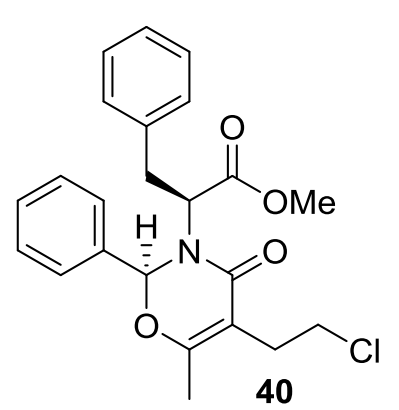

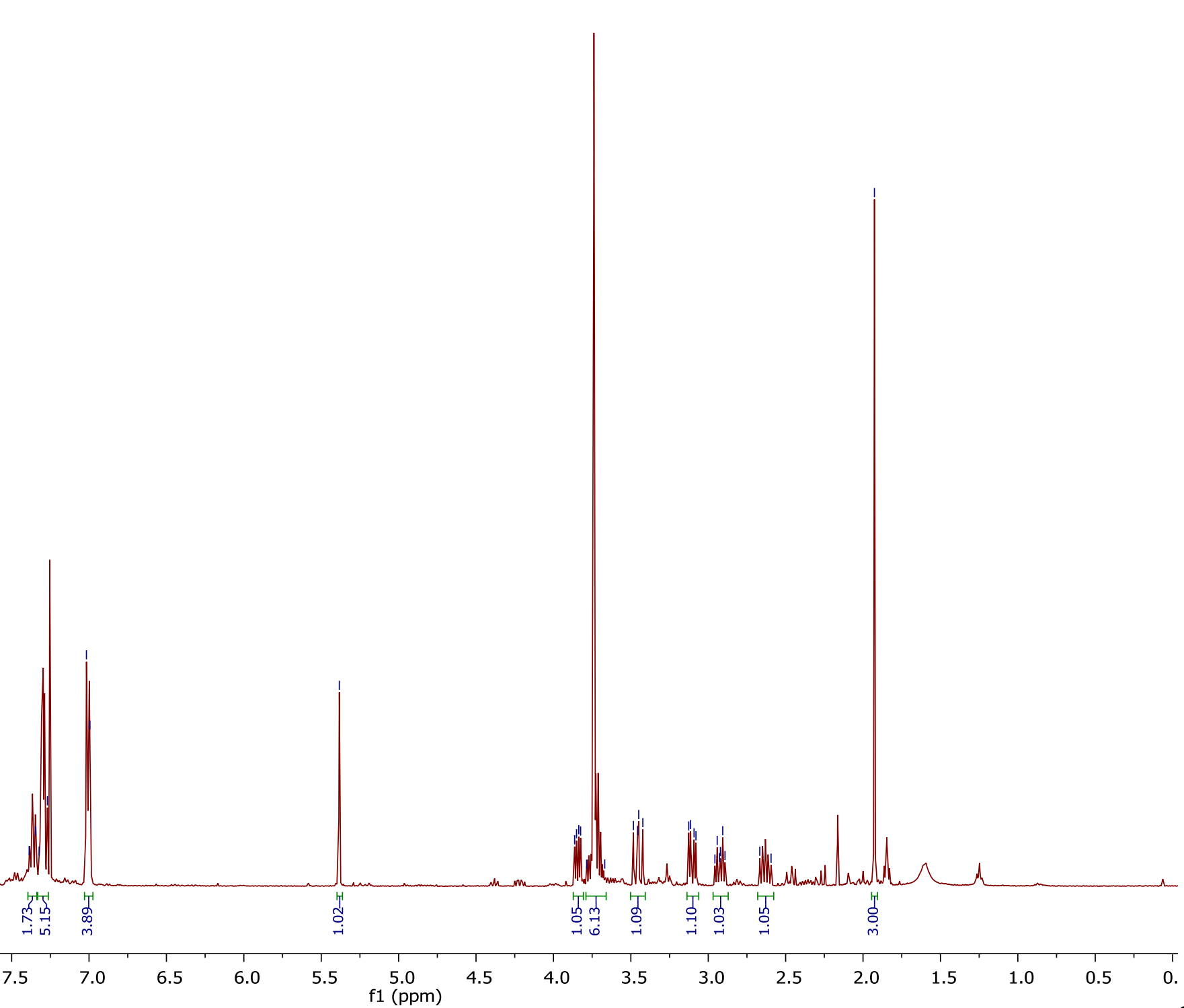




$$
\text { I. }
$$



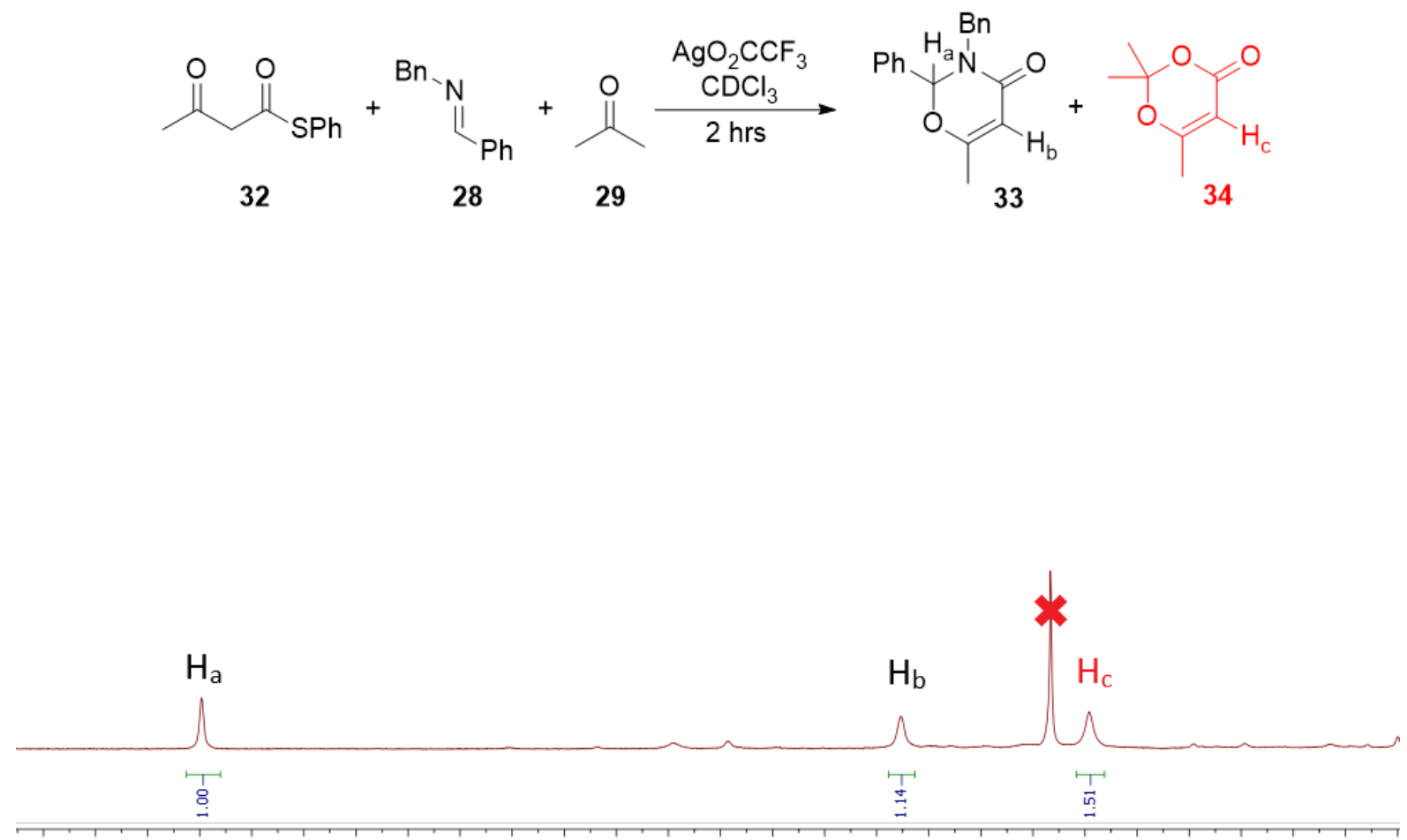

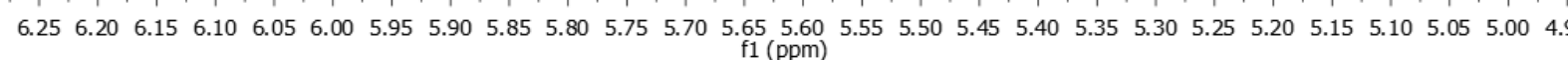

Figure S1. ${ }^{1} \mathrm{H}$ NMR spectrum $\left(400 \mathrm{MHz}, \mathrm{CDCl}_{3}, 298 \mathrm{~K}\right)$ of the reaction mixture for the ketone vs imine trapping experiment. 